\title{
Snow avalanche friction relation based on extended kinetic theory
}

\author{
Matthias Rauter $^{1,2}$, Jan-Thomas Fischer ${ }^{2}$, Wolfgang Fellin ${ }^{1}$, and Andreas Kofler ${ }^{2}$ \\ ${ }^{1}$ University of Innsbruck, Institute of Infrastructure, Division of Geotechnical and Tunnel Engineering, Innsbruck, Austria \\ ${ }^{2}$ Department of Natural Hazards, Austrian Research Centre for Forests (BFW), Innsbruck, Austria
}

Correspondence to: Matthias Rauter (matthias.rauter@uibk.ac.at)

Received: 23 March 2016 - Published in Nat. Hazards Earth Syst. Sci. Discuss.: 1 April 2016

Revised: 22 September 2016 - Accepted: 22 September 2016 - Published: 3 November 2016

\begin{abstract}
Rheological models for granular materials play an important role in the numerical simulation of dry dense snow avalanches. This article describes the application of a physically based model from the field of kinetic theory to snow avalanche simulations. The fundamental structure of the so-called extended kinetic theory is outlined and the decisive model behavior for avalanches is identified. A simplified relation, covering the basic features of the extended kinetic theory, is developed and implemented into an operational avalanche simulation software. To test the obtained friction relation, simulation results are compared to velocity and runout observations of avalanches, recorded from different field tests. As reference we utilize a classic phenomenological friction relation, which is commonly applied for hazard estimation. The quantitative comparison is based on the combination of normalized residuals of different observation variables in order to take into account the quality of the simulations in various regards. It is demonstrated that the extended kinetic theory provides a physically based explanation for the structure of phenomenological friction relations. The friction relation derived with the help of the extended kinetic theory shows advantages to the classic phenomenological friction, in particular when different events and various observation variables are investigated.
\end{abstract}

\section{Introduction}

Within the past few decades several software tools for the simulation of snow avalanches or, generally speaking, shallow granular flows have been developed, such as SamosAT (Sampl and Zwinger, 2004; Zwinger et al., 2003), TITAN2D (Pitman et al., 2003; Patra et al., 2005), RAMMS (Christen et al., 2010) or r.avaflow (Mergili et al., 2012). In this study the software SamosAT is utilized. The implemented flow model therein is the Savage-Hutter model (Savage and Hutter, 1989, 1991), which is related to the shallow water or Saint-Venant equations (de Saint-Venant, 1871). These models idealize avalanches and other free surface flows as depthaveraged flows. The Saint-Venant equations are set up in a Cartesian coordinate system and the normal stresses are assumed to be hydrostatic. In contrast, the Savage-Hutter equations are set up in a curvilinear coordinate system (compare, e.g., Bouchut and Westdickenberg, 2004) and the hydrostatic pressure assumption is replaced by an assumption for the lateral active or passive earth pressure, common in soil mechanics. The density is assumed to be constant in both models.

Within this framework rheological models attract a significant portion of attention. A widespread, classic phenomenological rheological model still utilized in depth-averaged models is the Voellmy friction relation (Voellmy, 1955). A motivation for this phenomenological friction relation, based on a physical model following Bagnold $(1954 ; 1966)$, is presented by Salm (1993). The underlying model of Bagnold (1954) represents a specialization of kinetic theory (Mitarai and Nakanishi, 2005; Lee and Huang, 2010). Another approach is presented by Issler and Gauer (2008), who apply the Norem-Irgens-Schieldrop model (Norem et al., 1987) to establish a rheological model for snow avalanches, yielding a similar relation as in this work. More recently, Buser and Bartelt (2009) introduced the concept of random kinetic energy to snow avalanche simulations. The energy evolution is described by a transport equation and influences the frictional behavior along the avalanche path. This approach shows some similarities to the kinetic theory model used in this work.

The rheology of granular materials has been investigated in many scientific works within the framework of three- 
dimensional continuum mechanics. An important category of microrheological models, dealing with rapid granular flows, is the kinetic theory (Campbell, 1990; Goldhirsch, 2003). Standard kinetic theory struggles to describe the dense flow regime at low shear rates. Recently developed extensions aim to take into account the formation of clusters (Jenkins, 2006, 2007) and enduring force chains (Berzi et al., 2011; Vescovi et al., 2013). The basis of the presented approach is the extended kinetic theory, as formulated by Vescovi et al. (2013). This microrheological model deals with both the quasi-static regime, described by the critical state theory, and the rapid, collisional flow, described by the kinetic theory of granular gases.

To implement the constitutive model into the depthaveraged dynamic model, several assumptions about the vertical structure of the flow are made, yielding a simplified friction relation. It is shown that the simplified expression is similar to classic friction relations. In a further step the obtained relation is compared to classic friction relations which are often applied in natural hazard estimation. For this task, back calculations of well-documented avalanches are conducted and minimal residuals in multiple observation variables, such as runout distance, affected area and velocity, are determined. It is shown that the relation obtained by kinetic theory allows to reduce the residuals for the presented events.

\section{Constitutive relations in the Savage-Hutter model}

The governing equations of the Savage-Hutter model, extended for entrainment, as implemented in SamosAT, for an incompressible, granular flow over a one-dimensional terrain can be expressed as

$$
\begin{aligned}
& \frac{\partial h}{\partial t}+\frac{\partial(h \bar{u})}{\partial x}=\frac{\dot{q}}{\rho}, \\
& \frac{\partial(h \bar{u})}{\partial t}+\frac{\partial\left(h \bar{u}^{2}\right)}{\partial x}=h g_{x}-\frac{1}{2 \rho} \frac{\partial\left(K_{\mathrm{a} / \mathrm{p}} \sigma_{\mathrm{b}} h\right)}{\partial x}-\frac{\tau_{\mathrm{b}}}{\rho} .
\end{aligned}
$$

These expressions can also be written for the twodimensional case (e.g., Sampl and Zwinger, 2004). Equation (1) describes the conservation of mass and Eq. (2) the conservation of momentum parallel to the slope surface. $x$ is the curvilinear coordinate, $z$ the coordinate perpendicular to $x$ and $t$ the time. $\rho$ represents the flow density, assumed to be constant, $h$ the slope perpendicular flow depth, $\bar{u}$ the slope parallel, depth-integrated velocity in $x$ direction, $\frac{1}{h} \int_{0}^{h} u_{x} \mathrm{~d} z$ and $g_{x}$ and $g_{z}$ are the gravitational accelerations in $x$ and $z$ direction, respectively. The entrainment rate $\dot{q}$ represents the mass entrained by the avalanche within a specific amount of time and area (with unit $\mathrm{kg} \mathrm{m}^{-2} \mathrm{~s}^{-1}$ ). Note that Eqs. (1) and (2) are only valid for small curvatures.

The resistance of the material against its deformation is considered with the second and third term on the right-hand side of Eq. (2). The second term represents the slope parallel stress gradient $\frac{\partial \sigma_{x}}{\partial x}$, expressed by the basal normal stress $\sigma_{\mathrm{b}}$ and the earth pressure coefficient $K_{\mathrm{a} / \mathrm{p}} . \sigma_{\mathrm{b}}$ is calculated with respect to the centripetal acceleration due to the basal curvature $\kappa$ as (Sampl and Zwinger, 2004; Fischer et al., 2012)

$\sigma_{\mathrm{b}}=\rho h\left(g_{z}+\kappa \bar{u}^{2}\right)$.

The earth pressure coefficient $K_{\mathrm{a} / \mathrm{p}}$ is given as

$K_{\mathrm{a} / \mathrm{p}}=\left\{\begin{array}{ll}K_{\mathrm{a}}=2 \frac{1-\sqrt{1-\cos ^{2} \phi / \cos ^{2} \delta}}{\cos ^{2} \phi}-1 & \text { if } \frac{\partial \bar{u}}{\partial x} \geq 0 \\ K_{\mathrm{p}}=2 \frac{1+\sqrt{1-\cos ^{2} \phi / \cos ^{2} \delta}}{\cos ^{2} \phi}-1 & \text { otherwise }\end{array}\right.$,

following a Mohr-Coulomb yield criterion (Savage and Hutter, 1989, 1991). Another approach by Salm (1966, 1993) is based on Rankine's earth pressure. Here, $\phi$ is the internal friction angle and $\delta$ is the bed friction angle. $K_{\mathrm{a} / \mathrm{p}}=1$ coincides with hydrostatic pressure and the Saint-Venant assumption and is a commonly used simplification (Christen et al., 2010). Bartelt et al. (1999) showed that the sensitivity of the relevant simulation results to the internal friction angle is rather small for snow avalanches. A detailed analysis is not in the scope of this work and a fixed internal friction angle, employing Eq. (4) is utilized.

The third term on the right-hand side of Eq. (2) describes the basal friction. Usually this term is a combination of a Coulomb type friction, $\sigma_{\mathrm{b}} \tan \delta$ and a velocity-dependent drag term, $f\left(\sigma_{\mathrm{b}}, \bar{u}\right) \bar{u}^{2}$ (Hutter et al., 2005).

\section{Constitutive model}

In this section we outline the fundamental structure of the extended kinetic theory for simple shear conditions. Classic kinetic theories are based on the statistical description of binary particle collisions, which are the governing processes at low volume fractions. The extended kinetic theory used here includes extensions (Jenkins, 2006, 2007; Berzi et al., 2011; Vescovi et al., 2013) to make the model suitable for high volume fractions and therefore dense flows. In this section we employ material parameters for idealized quartz sand to identify the basic features of the extended kinetic theory. In Sect. 6.5 we give estimations for material parameters suitable for snow, based on back calculations of real avalanche events.

\subsection{Extended kinetic theory}

The extended kinetic theory as formulated by Vescovi et al. (2013) combines two theories to model granular flows from low to high velocities, namely the kinetic theory and the critical state theory from soil mechanics (Roscoe et al., 1958; Schofield and Wroth, 1968), which are commonly accepted to hold for granular media. This theory is proven to deliver good results for various physical experiments with granules (Vescovi, 2014). Note that there exists a wide range of kinetic 


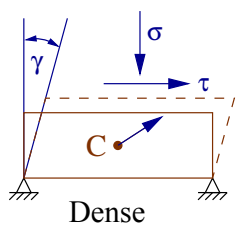

(a)

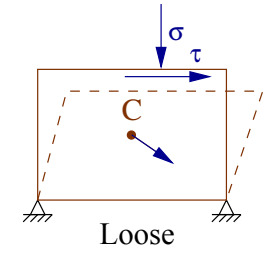

(b)

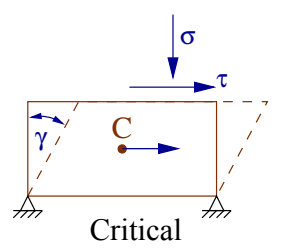

(c)
Figure 1. Simple shear test: $\mathrm{C}$ denotes the center of gravity with an arrow showing the direction of its displacement during shearing.

theories (e.g., Garzó and Dufty, 2002; Serero et al., 2006; Sela and Goldhirsch, 1998; Brey et al., 1998; Zamankhan, 1995; Alam et al., 2002; Willits and Arnarson, 1999; Jenkins and Mancini, 1987, 1989; Rahaman et al., 2003; Arnarson and Willits, 1998; Garzó and Dufty, 1999; Bartelt et al., 2006) and extensions (e.g., Goldshtein and Shapiro, 1995; Jenkins and Richman, 1986; Jenkins and Zhang, 2002; Lun, 1991; Lun and Savage, 1987; Brilliantov and Pöschel, 2001; Pöschel and Buchholtz, 1995; Lois et al., 2005, 2006; Mills et al., 2008; Mitarai and Nakanishi, 2007; Jenkins, 2006, 2007; Vescovi et al., 2013), which differ in various ways from the kinetic theory used here; see, e.g., the discussion in Vescovi (2014) and Goldhirsch (2003) for models related to granular flow.

\subsubsection{Critical state theory}

The critical state theory was developed in soil mechanics, where the deformation rates are very small and therefore no inertial forces occur. This theory can be outlined with the help of the simple shear test with constant normal stress $\dot{\sigma}=0$, which is similar to the situation sketched in Fig. 1 . A soil sample is sheared by an increasing shear angle $\gamma$. The sample has an initial density, and with that an initial concentration $v_{0}=V_{\mathrm{p}} / V$, with the volume of the particles $V_{\mathrm{p}}$ and the total volume of the sample $V$. Note that in soil mechanics it is more common to use the void ratio $e=(1-v) / v$. The shear stress increases due to the imposed shearing. An initially dense sample increases its total volume (see Fig. 1a) and an initially loose sample decreases its volume (see Fig. 1b). However, the concentration of both samples of the same material approach the same value, the so-called critical concentration $v_{\mathrm{ss}}$ and this concentration remains constant for further shearing (see Fig. 1c), i.e., $\dot{v}=0$. At this stage the shear stress remains constant too, i.e., $\dot{\tau}=0$. The state $\dot{\sigma}=\dot{\tau}=\dot{v}=0$ for $\dot{\gamma} \neq 0$ is called critical state or steady state.

The shear stress at critical state is related to the applied normal stress: $\tau=\sigma \tan \phi_{\mathrm{ss}}^{\prime}$, with the critical (steady state) friction angle $\phi_{\mathrm{ss}}^{\prime}$. The critical concentration depends on the applied normal stress $\sigma$, i.e., for tests with a higher normal stress a larger critical concentration (a higher critical density) will be reached, which constitutes a relation between normal stress and concentration at critical state. In turn the normal stress can be regarded as function of the critical concentration: $\sigma\left(v_{\mathrm{ss}}\right)$. It is important to know one limiting value of this relation: the concentration of any granular media at rest cannot be less than a minimal concentration $v_{\mathrm{rlp}}$ (random loose package or maximal void ratio $e_{\max }$ in soil mechanics), depending on the material. The critical concentration is bounded by this limit. Physical experiments to determine this limit are performed at very low stress levels, virtually zero stress level, and the relation between the normal stress and the concentration at critical state has to provide $\sigma=0$ for $v_{\mathrm{sS}}=v_{\mathrm{rlp}}$. Note that $\tau=0$ in this limiting state, too. Since the shearing in any flowing mass is high, it can be assumed that the critical state is reached (compare Figs. 1c and 2). With this assumption, the concentration in the flow is $v=v_{\mathrm{ss}}$, and we will drop the index ss in the following.

\subsubsection{Kinetic theory}

The second constituent of the applied theory is the kinetic theory, which provides relations for the normal and shear stress based on statistical considerations of the collisions between particles in the volume $V$ for high shear rates $\dot{\gamma}$. It is reasonable to assume that the particle collisions will lead to high stresses for high shear rates and high concentrations. Appropriate relations can be established to provide both stresses $\sigma_{\mathrm{c}}(\nu, \dot{\gamma})$ and $\tau_{\mathrm{c}}(\nu, \dot{\gamma})$ as functions of the concentration and the shear rate. These functions are very complex and will be given in the next section. So far, it is sufficient to keep the qualitative behavior in mind: $\sigma_{\mathrm{c}}$ and $\tau_{\mathrm{c}}$ will approach zero for a vanishing shear rate and will decrease for a decreasing concentration.

The main idea of the extended kinetic theory by Vescovi et al. (2013) is to combine the quasi-static stresses for vanishing shear rates at critical state (denoted by q) with the collisional stresses (denoted by c) of the kinetic theory:

$$
\begin{aligned}
& \sigma=\sigma_{\mathrm{q}}(\nu)+\sigma_{\mathrm{c}}(\nu, \dot{\gamma}), \\
& \tau=\sigma_{\mathrm{q}}(\nu) \tan \phi_{\mathrm{ss}}^{\prime}+\tau_{\mathrm{c}}(\nu, \dot{\gamma}) .
\end{aligned}
$$

It is important to note that the concentration is the critical concentration in both stress contributions. For concentrations lower than the loosest quasi-static limit $v_{\mathrm{rlp}}$, the quasi-static stresses will vanish, and for a vanishing shear rate the collisional stresses will vanish. As such, critical state behavior will be ensured for low shear rates and purely collisional behavior will be ensured for densities lower than any statically possible density (which is not considered as dense flow herein). At the bottom, the normal stress $\sigma$ (Eq. 5) has to be in equilibrium with the basal normal stress $\sigma_{\mathrm{b}}$ (Eq. 3) of the flow model. This can be used to calculate the basal shear stress $\tau_{\mathrm{b}}$ as shown in Sect. 4. Equation (3) can be extended by an additional term, accounting for vertical acceleration due to dilatancy, called dispersive pressure (Buser and Bartelt, 2015; Bartelt and Buser, 2016; Bartelt et al., 2016), but is neglected 


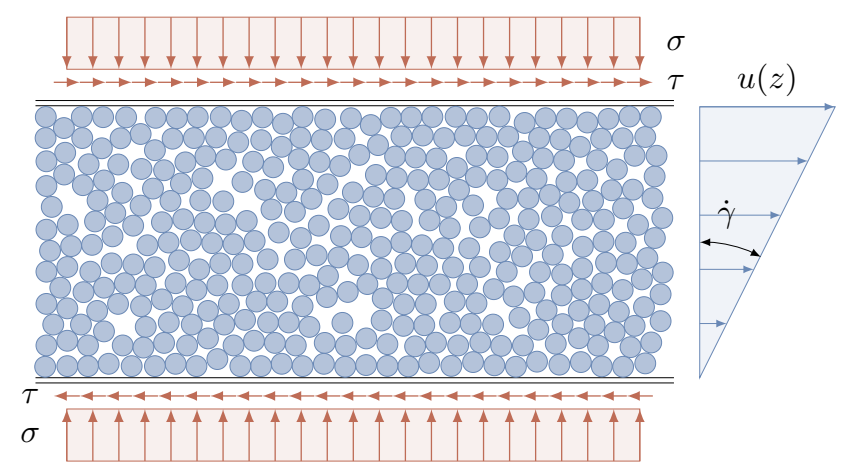

Figure 2. Simple shear flow setup with linear velocity profile $u(z)$. Flow variables included into the constitutive relation, e.g., the shear rate $\dot{\gamma}$, normal stress $\sigma$ and the shear stress $\tau$, are consequently constant.

in this work. Another approach to include dilatancy in depthaveraged granular flow models can be found in Iverson and George (2014).

\subsection{Model formulation}

The simple shear setup, on which the presented model has been developed, is shown in Fig. 2. In this setup, the relevant stresses and deformations are constant over the whole domain. Consequently, only local field values have an influence on the constitutive behavior. This matches arbitrary fields when zeroth-order expansions of spatial and temporal gradients are employed (e.g., Garzó and Dufty, 1999). Moreover, no diffusive or convective transport is present in this setup.

Flows of granular material can display a large span of grain concentrations. Microscopic mechanical processes and consequently the macroscopic behavior of the material changes substantially with the concentration or solid fraction $v$ and the granular temperature $T$. The concentration (or solid volume fraction) is statistically defined as

$$
v=\frac{\pi n d^{3}}{6}
$$

where $d$ is the particle diameter and $n$ the number of particles per unit volume at position $\mathbf{x}$ and time $t$, given as

$n(\mathbf{x}, t)=\int f(\mathbf{v}, \mathbf{x}, t) \mathrm{d} \mathbf{v}$,

where $f(\mathbf{v}, \mathbf{x}, t)$ is the single particle velocity distribution function, defined as the number of particles at position $\mathbf{x}$ with velocity $\mathbf{v}$ at time $t$. Consequently $f(\mathbf{v}, \mathbf{x}, t) / n(\mathbf{x}, t)$ is the probability density for a particle at position $\mathbf{x}$ and time $t$ to have the velocity v (e.g., Goldhirsch, 2003; Jenkins and Berzi, 2010). Two different approaches are used to derive $f(\mathbf{v}, \mathbf{x}, t)$, the Boltzmann equation and the Enskog equation (Garzó and Dufty, 1999; Vescovi, 2014). The granular tem- perature is associated with the fluctuation of the particle velocity,

$\frac{3}{2} T=\frac{1}{n} \int \frac{1}{2}(\mathbf{u}-\mathbf{v})^{2} f(\mathbf{v}, \mathbf{x}, t) \mathrm{d} \mathbf{v}$,

where the mean velocity $\mathbf{u}$ is defined as

$\mathbf{u}=\frac{1}{n} \int \mathbf{v} f(\mathbf{v}, \mathbf{x}, t) \mathrm{d} \mathbf{v}$.

Although the rheological model assumes simple shear conditions, the fluctuating velocity is three-dimensional, leading to the factor $3 / 2$ in Eq. (9).

Concentration and granular temperature are determined by the hydrodynamic fields and their gradients. For the presented simple shear setup, these are the normal stress along the transversal direction $\sigma$ and the shear rate $\dot{\gamma}$, given as the derivative of the velocity in its perpendicular direction, $\frac{\partial u}{\partial z}$ (see Fig. 2).

To describe the whole range of flow configurations, multiple mechanical processes, described by different theories, need to be taken into account (Berzi et al., 2011; Vescovi et al., 2013).

On the one hand, the critical state theory (Roscoe et al., 1958; Schofield and Wroth, 1968) describes granular material at vanishingly small shear rates $\dot{\gamma}$. This model is completely time independent and does not take into account the velocity of any process. The stresses in the material are based completely on enduring force chains between particles. Also the assumption of the incompressibility of granular flows follows from this theory: granular material under motion always reaches asymptotically a certain stress dependent concentration, the critical concentration. The contribution of quasistatic stresses, e.g., force chains, to the total stresses, as described by the critical state theory is given as

$\sigma_{\mathrm{q}}=f_{0} \frac{K}{d}$,

$\tau_{\mathrm{q}}=\sigma_{\mathrm{q}} \tan \phi_{\mathrm{ss}}^{\prime}$,

with

$f_{0}= \begin{cases}a \frac{v-v_{\mathrm{rlp}}}{v_{\mathrm{s}}-v} & \text { if and only if } v>v_{\mathrm{rlp}} \text { and } v_{\mathrm{rlp}}<v_{\mathrm{s}} \\ 0 & \text { otherwise }\end{cases}$

$K=\frac{\pi d E}{8}$.

Equations (11) and (12) can be considered as critical state line in the $\nu-\sigma-\tau$ space. Material parameters are the tangent of the internal friction angle at the critical state $\tan \phi_{\mathrm{ss}}^{\prime}$, the Young's modulus of the particles $E$, the particle diameter $d$, the concentration at random loose packing $v_{\text {rlp }}$, the concentration at closest packing $v_{\mathrm{s}}$ and the dimensionless parameter $a$.

On the other hand, the kinetic theory of granular gases describes the granular material under the influence of high shear rates. The stresses in the material are based on short 
contacts between particles, i.e., elastoplastic collisions. The following form of this theory was proposed by Garzó and Dufty (1999) and extended by Jenkins and Berzi (2010) and Vescovi et al. (2013). It is limited to homogeneous, steady, simple shear flows of identical, dry, spherical particles.

The contribution from collisions to the total stresses is given as

$\sigma_{\mathrm{c}}=\rho_{\mathrm{p}} f_{1} f_{4} T$

$\tau_{\mathrm{c}}=\rho_{\mathrm{p}} d f_{2} f_{4} T^{1 / 2} \dot{\gamma}$,

and the dissipation rate of the granular temperature is given as

$\Gamma_{\mathrm{c}}=\rho_{\mathrm{p}} \frac{f_{3}}{L} f_{4} T^{3 / 2}$,

with

$$
\begin{aligned}
f_{1} & =4 v G F, \\
f_{2} & =\frac{8 J}{5 \pi^{1 / 2}} v G, \\
f_{3} & =\frac{12}{\pi^{1 / 2}}\left(1-\epsilon^{2}\right) v G, \\
G & =v g_{0}, \\
g_{0} & = \begin{cases}\frac{2-v}{2(1-v)^{3}} & v \leq 0.49 \\
\frac{5.69\left(v_{\mathrm{s}}-0.49\right)}{v_{\mathrm{s}}-v} & v>0.49\end{cases} \\
F & =\frac{1+\epsilon}{2}+\frac{1}{4 G}, \\
J & =\frac{1+\epsilon}{2} \\
+ & \frac{\pi}{32} \frac{[5+2(1+\epsilon)(3 \epsilon-1) G][5+4(1+\epsilon) G]}{\left[24-6(1-\epsilon)^{2}-5\left(1-\epsilon^{2}\right)\right] G^{2}}, \\
f_{4} & =\left[1+2 \frac{d}{s}\left(\frac{\rho_{\mathrm{p}} T}{E}\right)^{1 / 2}\right]^{-1}, \\
s & =\frac{\sqrt{2}}{12} \frac{d}{G}, \\
f_{5} & =\frac{L}{d} \frac{f_{2}}{f_{3}}, \\
\frac{L}{d} & =\max \left[1,\left(\frac{c^{2} G^{2 / 3} f_{3}}{4 f_{2}}\right)^{1 / 3}\right] .
\end{aligned}
$$

Additional material parameters, introduced by the kinetic theory, are the particle density $\rho_{\mathrm{p}}$, the coefficient of restitution $\epsilon$ and the dimensionless parameter $c$. The parameter set is summarized in Table 1. The functions $f_{1}, f_{2}$ and $f_{3}$ are solely dependent on the concentration $v$ and the material parameters; the function $f_{4}$ takes into account the particle stiffness. $g_{0}$ is the radial distribution function, following the approach of Carnahan and Starling (1969) for $v \leq 0.49$ and the approach of Garzó and Dufty (1999) for $v>0.49$. $L$ is the correlation length, accounting for correlated motion of
Table 1. Material parameters of the extended kinetic theory.

\begin{tabular}{lll}
\hline Parameter & Description & Unit \\
\hline$\phi_{\mathrm{Ss}}^{\prime}$ & Critical friction angle & - \\
$d$ & Particle diameter & $\mathrm{m}$ \\
$K$ & Particle stiffness & Pa m \\
$a$ & Critical state line parameter & - \\
$v_{\mathrm{s}}$ & Dense packing concentration & - \\
$v_{\mathrm{rlp}}$ & Loose packing concentration & - \\
$\rho_{\mathrm{p}}$ & Particle density & $\mathrm{kg} \mathrm{m}^{-3}$ \\
$\epsilon$ & Coefficient of restitution & - \\
$c$ & Parameter for correlated motion & - \\
\hline
\end{tabular}

particles at high concentrations and $s$ is the mean separation distance among particles (i.e., the mean free path between collisions) (Vescovi et al., 2013).

The total stresses can be expressed as sum of the quasistatic and the collisional stresses, combining critical state theory and kinetic theory:

$$
\begin{aligned}
& \sigma=\sigma_{\mathrm{q}}+\sigma_{\mathrm{c}}=\frac{K}{d} f_{0}+\rho_{\mathrm{p}} f_{1} f_{4} T, \\
& \tau=\tau_{\mathrm{q}}+\tau_{\mathrm{c}}=\frac{K}{d} f_{0} \tan \phi_{\mathrm{ss}}^{\prime}+\rho_{\mathrm{p}} d f_{2} f_{4} T^{1 / 2} \dot{\gamma} .
\end{aligned}
$$

Coupling of quasi-static force chains and collisions is ensured by sharing the concentration (compare Eqs. 5 and 6). The concentration determines the dominant process, e.g., quasi-static force chains at high concentrations and collisions at low concentrations. Blending between those limit cases is based on the well-known concentration dependence of both processes. The evolution of the granular temperature is described by the conservation equation,

$\frac{3}{2} \rho \frac{\partial T}{\partial t}=\tau_{\mathrm{c}} \dot{\gamma}-\nabla \cdot \mathbf{q}_{\mathrm{c}}-\Gamma_{\mathrm{c}}$,

with production $\tau_{\mathrm{c}} \dot{\gamma}$, flux $\mathbf{q}_{\mathrm{c}}$ and dissipation $\Gamma_{\mathrm{c}}$. The assumptions of steady state and simple shear imply that granular temperature is dissipated where it has been produced. This approach, called equilibrium assumption (van Wachem, 2000), is also applied to dense flows apart from steady, simple shear conditions (Syamlal et al., 1993; Boemer et al., 1997; van Wachem et al., 1998, 1999; van Wachem, 2000) and is justified by the dominance of generation and dissipation terms over convection and diffusion terms. Other theories do not assume equilibrium; e.g., for a depth-averaged granular temperature transport equation, see Christen et al. (2010). With the equilibrium assumption, the transport equation can be reduced to an algebraic formulation of the equilibrium state, given as (Vescovi et al., 2013)

$\Gamma_{\mathrm{c}}=\tau_{\mathrm{c}} \dot{\gamma}$.

This assumption allows to apply the kinetic theory to operational simulation software without introducing additional differential equations. 


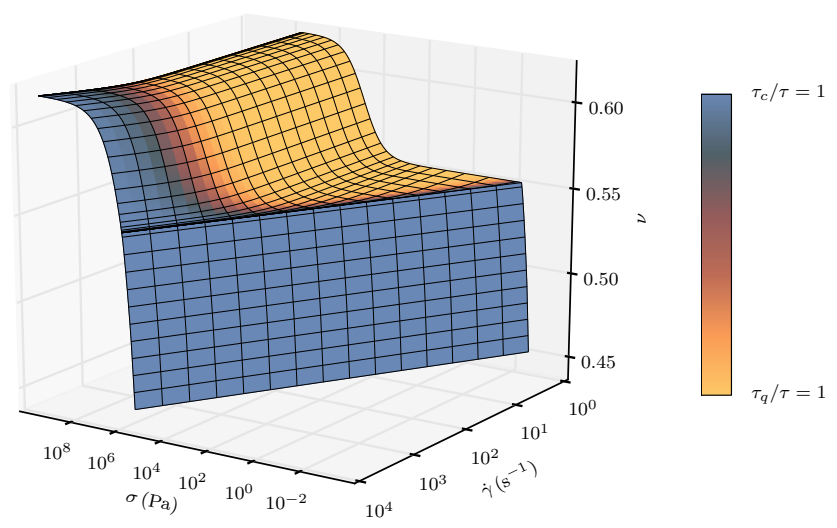

Figure 3. Critical state surface in the $\sigma-\dot{\gamma}-v$ space. The color marks the origin of stresses: in yellow areas frictional stresses are dominant and in blue areas collisional stresses.

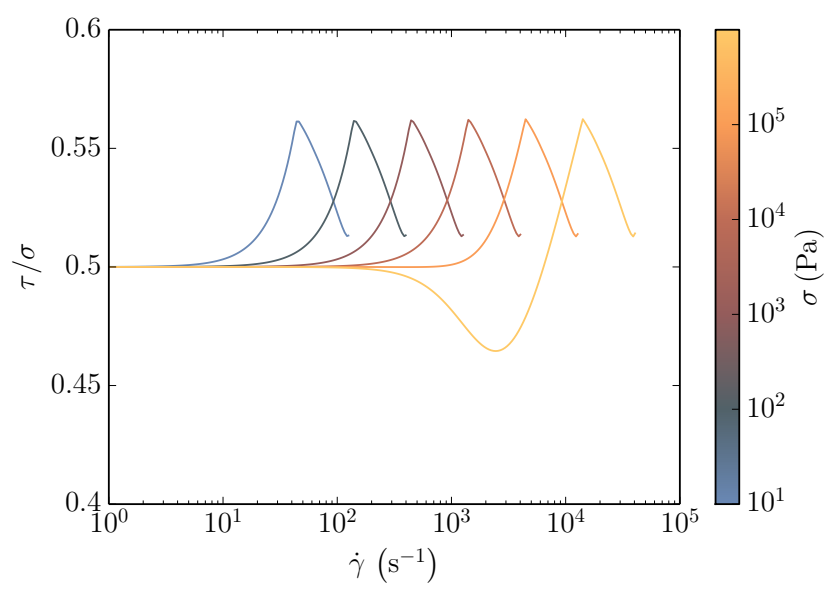

Figure 4. Stress ratio $\tau / \sigma$ in dependence of normal stress $\sigma$ and shear rate $\dot{\gamma}$.

By introducing Eqs. (17) and (18) into Eq. (33), the granular temperature can be expressed as a function of the shear rate $\dot{\gamma}$ and the concentration $v$ :

$T=d^{2} f_{5} \dot{\gamma}^{2}$.

Introducing Eq. (34) in Eqs. (30) and (31) leads to an expression for the total stresses, only depending on $\dot{\gamma}$ and $v$ :

$\sigma=\frac{K}{d} f_{0}+\rho_{\mathrm{p}} d^{2} f_{1} f_{4} f_{5} \dot{\gamma}^{2}$,

$\tau=\frac{K}{d} f_{0} \tan \phi_{\mathrm{ss}}^{\prime}+\rho_{\mathrm{p}} d^{2} f_{2} f_{4} f_{5}^{1 / 2} \dot{\gamma}^{2}$.

According to Eqs. (35) and (36), it is possible to characterize the flow regime with only two state variables. In the case of known values for $\sigma$ and $\dot{\gamma}$, Eq. (35) can be used to solve for $v$, using Newton-Raphson (e.g., Press et al., 1996) or another root-finding routine. $\tau$ can then be calculated with Eq. (36), $T$ with Eq. (34), if required.

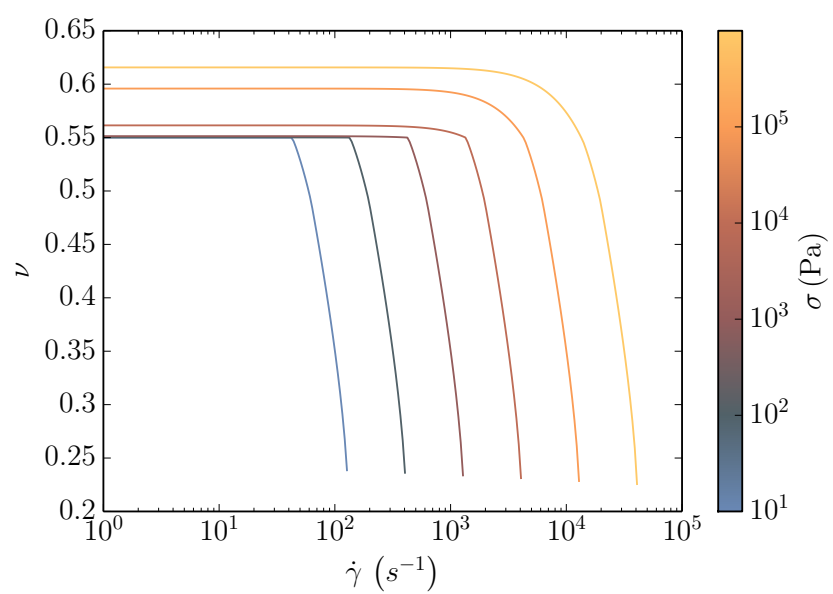

Figure 5. Concentration $v$ in dependence of normal stress $\sigma$ and shear rate $\dot{\gamma}$.

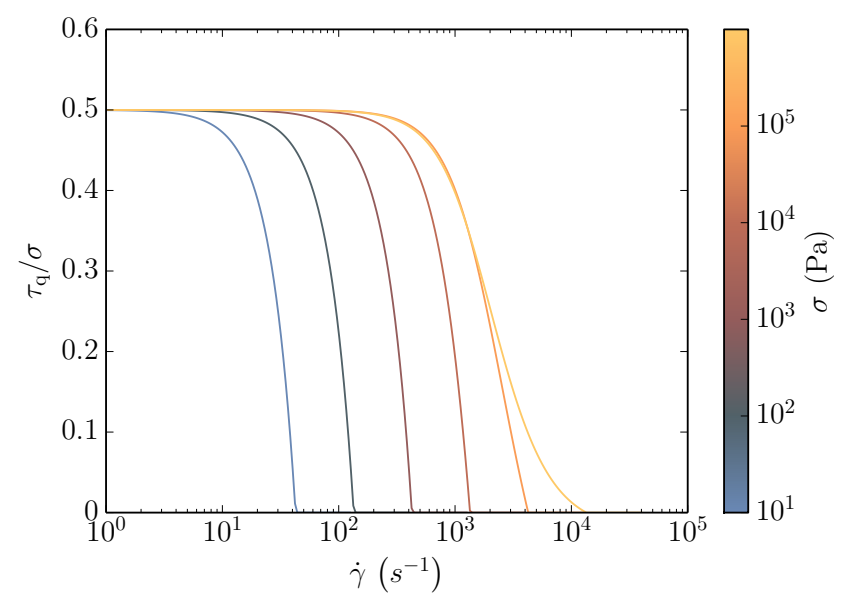

Figure 6. Contribution of enduring force chains: stress ratio $\tau_{\mathrm{q}} / \sigma$.

Material parameters for snow are not available at the current stage. However, they can be estimated from the results of back calculations of real-scale avalanches, conducted in the second part of this paper. To qualitatively highlight the most important features of the constitutive model, it is analyzed for an idealized $1 \mathrm{~mm}$ quartz sand $(d=$ $1 \mathrm{~mm}, \rho_{\mathrm{p}}=2600 \mathrm{~kg} \mathrm{~m}^{-3}, K=2.8 \times 10^{7} \mathrm{~Pa} \mathrm{~m}, \epsilon=0.6, c=$ $0.5, a=1.8 \times 10^{-6}, \tan \phi_{\mathrm{ss}}^{\prime}=0.5, v_{\mathrm{s}}=0.619, v_{\mathrm{rlp}}=0.55$; Vescovi et al., 2013, see Figs. 3-10). Basic features identified in this section will be approximated with a simplified relation to reduce the amount of material parameters, increase the numerical stability and reduce the computational effort.

Figure 3 shows the dynamic critical state surface in the $\sigma-$ $\dot{\gamma}-v$ space. According to the presented theory, flow states are limited to this surface. The color in Fig. 3 shows the dominant source of stresses, which can be interpreted as flow regime. In yellow areas, enduring contacts, forming elastic networks between particles, are dominant (referred to as quasi-static regime in da Cruz et al., 2005; Berzi et al., 2011; 


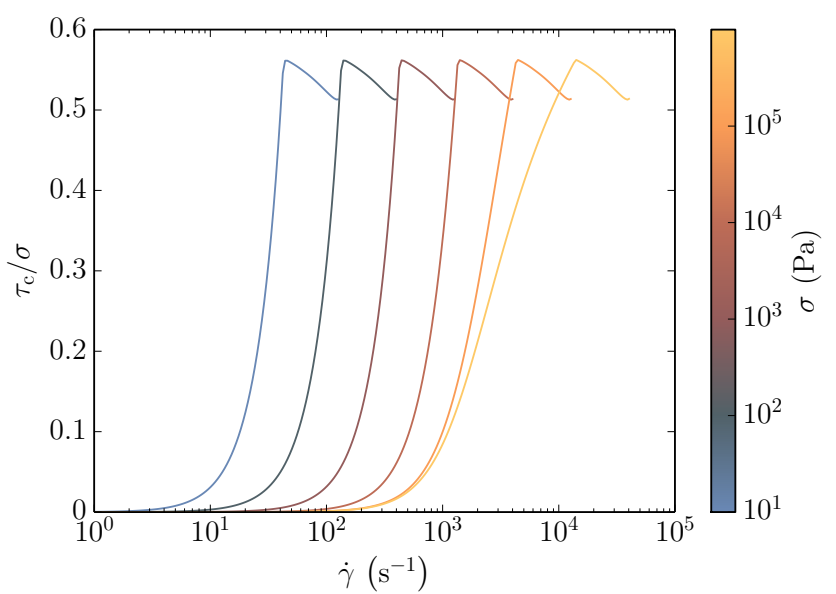

Figure 7. Contribution of collisions: stress ratio $\tau_{\mathrm{c}} / \sigma$.

Vescovi et al., 2013, or elastic-quasi-static regime in Campbell, 2002, 2005, 2006). In blue areas, collisional stresses are dominant (referred to as collisional regime in da Cruz et al., 2005; Vescovi et al., 2013; Berzi et al., 2011, kinetic regime in da Cruz et al., 2005; Forterre and Pouliquen, 2008; Vescovi et al., 2013; Berzi et al., 2011, or inertial-collisional regime in Campbell, 2002, 2005, 2006). The flow is purely collisional for concentrations below the random loose package: $v<v_{\text {rlp }}$. The red area represents a transitional zone between those cases, where both effects co-exist (referred to as dense regime in da Cruz et al., 2005, elastic-inertial regime in Campbell, 2002, 2005, 2006, or intermediate regime in Vescovi, 2014).

The granular temperature, which is not shown, is almost solely dependent on the shear rate $\dot{\gamma}$.

The stress ratio $\tau / \sigma$ and the respective concentration $v$ for a given set of $\sigma$ and $\dot{\gamma}$ are shown in Figs. 4 and 5. The constitutive model predicts a stress ratio $\tau / \sigma=\tan \phi_{\mathrm{ss}}^{\prime}$ for $\dot{\gamma} \rightarrow 0$, corresponding to critical state theory and a stress ratio corresponding to kinetic theory for high shear rates. The concentration is almost unaffected by changes in shear rate $\dot{\gamma}$ until it drops below $v_{\text {rlp }}$, e.g., at $\dot{\gamma} \approx 5 \times 10^{2} \mathrm{~s}^{-1}$ for $\sigma=10^{3} \mathrm{~Pa}$. At this point the concentration decreases abruptly and the shear stress lowers with increasing shear rate, after reaching its peak. This behavior can be interpreted as a transition from a dense flow to a powder cloud like flow. This work is focused on dense flow - the post-peak behavior is not investigated.

A separation of quasi-static and collisional stresses is shown in Figs. 6 and 7. For small stress levels, increasing collisional stresses $\tau_{\mathrm{c}}$ can compensate the decreasing quasistatic stresses $\tau_{\mathrm{q}}$. At high stress levels, $\sigma>\sigma^{*}\left(\nu>v^{*}\right)$, this is not the case and the stress ratio $\frac{\tau}{\sigma}=\frac{\tau_{\mathrm{q}}+\tau_{\mathrm{c}}}{\sigma}$ shows a nonmonotonic behavior before reaching the peak (Fig. 4). The thresholds for the non-monotonic behavior, $\sigma^{*}$ and $\nu^{*}$ can be calculated as (Vescovi et al., 2013)

$$
\begin{aligned}
\sigma^{*} & =a \frac{v^{*}-v_{\mathrm{rlp}}}{v_{\mathrm{s}}-v^{*}} \frac{K}{d}, \\
v^{*} & =v_{\mathrm{s}} \frac{\mathcal{B}^{9}\left(\tan \phi_{\mathrm{ss}}^{\prime}\right)^{-9}}{\mathcal{B}^{9}\left(\tan \phi_{\mathrm{ss}}^{\prime}\right)^{-9}+5.69\left(v_{\mathrm{s}}-0.49\right)},
\end{aligned}
$$

where

$\mathcal{B}=\left(\frac{48}{5 \pi(1+\epsilon)^{2}}\right)^{1 / 2}\left(\frac{\left(1-\epsilon^{2}\right)^{2} J^{4}}{15 c^{2}}\right)^{1 / 6}$

In the following, we focus on the monotonic dense flow regime, where $v_{\text {rlp }} \leq v \leq v^{*}$.

It is not feasible to implement the complete extended kinetic theory model in an operational simulation tool. The main reason is that for certain combinations of normal stress $\sigma$ and shear rate $\dot{\gamma}$, no solution for the concentration $v$ can be obtained (e.g., white space in Fig. 10), which makes the numerical treatment of the whole flow model unstable. Moreover, the procedure to solve the constitutive equations requires an iterative root-finding method, which is computational expensive. Another difficulty arises because the velocity profile is required to link the shear-rate-dependent rheology model with the depth-integrated flow model. The velocity profile cannot be calculated analytically for the complex kinetic theory model. Numerical simulations of the velocity profile, employing the full extended kinetic theory can be found in Rauter (2015). In this work we stick to the analytical description of the velocity profile. Because of these reasons, approximations of Eqs. (35) and (36) are made. In analogy to other studies (see Ancey, 2007, for a review) two approaches, varying the separation of friction into two parts, are evaluated. The first approach separates friction by their source (quasi-static-collisional), while the second approach separates friction into a shear-rate-independent and shearrate-dependent part. The first approach is given as

$\tau=\mu(\sigma, \dot{\gamma}) \sigma+\tilde{\lambda}(\sigma, \dot{\gamma}) \dot{\gamma}^{2}$,

with

$\mu(\sigma, \dot{\gamma})=\tan \phi_{\mathrm{ss}}^{\prime} f_{0}$,

$\tilde{\lambda}(\sigma, \dot{\gamma})=\rho_{\mathrm{p}} d^{2} f_{2} f_{4} f_{5}^{1 / 2}$.

Within this formulation, $\tilde{\lambda} \dot{\gamma}^{2}$ represents solely collisional stresses. The decrease of quasi-static stresses is considered with the share rate dependence of $\mu$.

The second approach is given as

$\tau=\tan \phi_{\mathrm{ss}}^{\prime} \sigma+\lambda(\sigma, \dot{\gamma}) \dot{\gamma}^{2}$

with

$\lambda(\sigma, \dot{\gamma})=\rho_{\mathrm{p}} d^{2} f_{4} f_{5}^{1 / 2}\left(f_{2}-f_{1} f_{5}^{1 / 2} \tan \phi_{\mathrm{ss}}^{\prime}\right)$. 


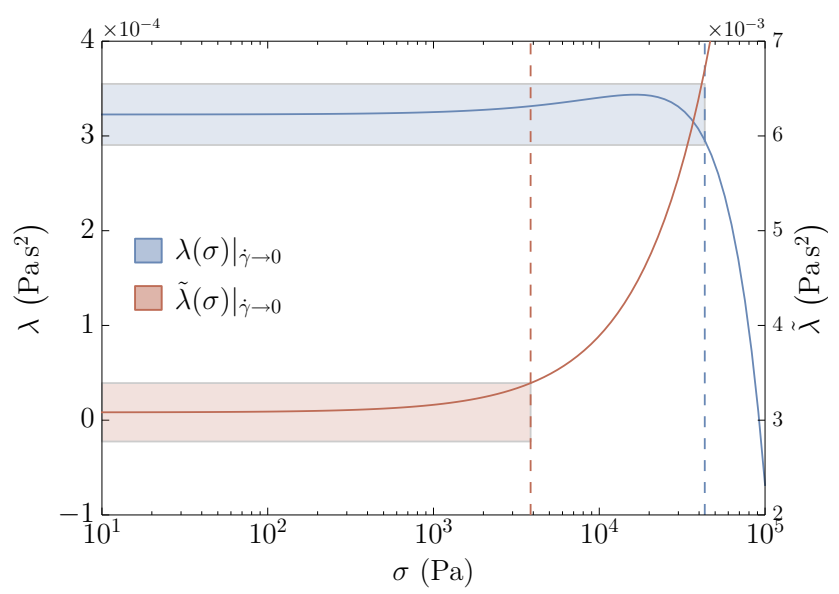

Figure 8. The factors $\lambda$ (blue) and $\tilde{\lambda}$ (red) for $\dot{\gamma} \rightarrow 0$. The colored areas show ranges of $\sigma$ where the respective value can be approximated with the value at $\sigma \rightarrow 0$ within an error of $\pm 10 \%$. $\lambda$ remains in the range of $\left.\lambda\right|_{\sigma \rightarrow 0} \pm 10 \%$ up to $4 \times 10^{4} \mathrm{~Pa}, \tilde{\lambda}$ in the range of $\left.\widetilde{\lambda}\right|_{\sigma \rightarrow 0} \pm 10 \%$ up to $4 \times 10^{3} \mathrm{~Pa}$.

Here, the term $\lambda \dot{\gamma}^{2}$ also accounts for the decreasing quasistatic stress. Both $\tilde{\lambda}$ and $\lambda$ are approximately constant within a certain range of $\sigma$ and $\dot{\gamma}$. The second approach with constant $\mu=\tan \phi_{\mathrm{sS}}^{\prime}$ leads to a better approximation (see Fig. 8) and a simpler formulation with less parameters. However, the non-monotonic behavior at high stress levels cannot be reproduced with this approach. Values for $\lambda$ are shown in Fig. 10. Up to a normal stress of $10^{4} \mathrm{~Pa}$ and until the peak is reached, $\lambda$ can be approximated as constant. The resulting simplified relation is shown and compared to the full kinetic theory in Fig. 9. Thus, Eq. (43) with a constant value for $\lambda$ is employed in the following:

$\tau=\mu \sigma+\lambda \dot{\gamma}^{2}$.

\section{Velocity profile and kinematic relations}

The constitutive model obtained by the granular kinetic theory results in a relation depending on the shear rate, which does not explicitly appear in depth-averaged models. However, the equilibrium of stresses at the bottom of the avalanche requires that

$\tau_{\mathrm{b}}=\tau\left(\sigma_{\mathrm{b}}, \dot{\gamma}_{\mathrm{b}}\right)$,

where $\sigma_{\mathrm{b}}$ is the normal stress at the bottom and $\dot{\gamma}_{\mathrm{b}}$ is the shear rate at the bottom. According to the Savage-Hutter model and related friction models, the friction may depend on velocity. To obtain an expression of the form $\tau_{\mathrm{b}}(\bar{u}, h)$ we need to express $\sigma_{\mathrm{b}}$ (see Eq. 3) and $\dot{\gamma}_{\mathrm{b}}$ with known flow variables. Therefore a reconstruction of the velocity profile is required.

Supposing that the avalanche has reached its steady state on a slope with constant inclination $\alpha$ and that $\frac{\partial h}{\partial x}$ is very

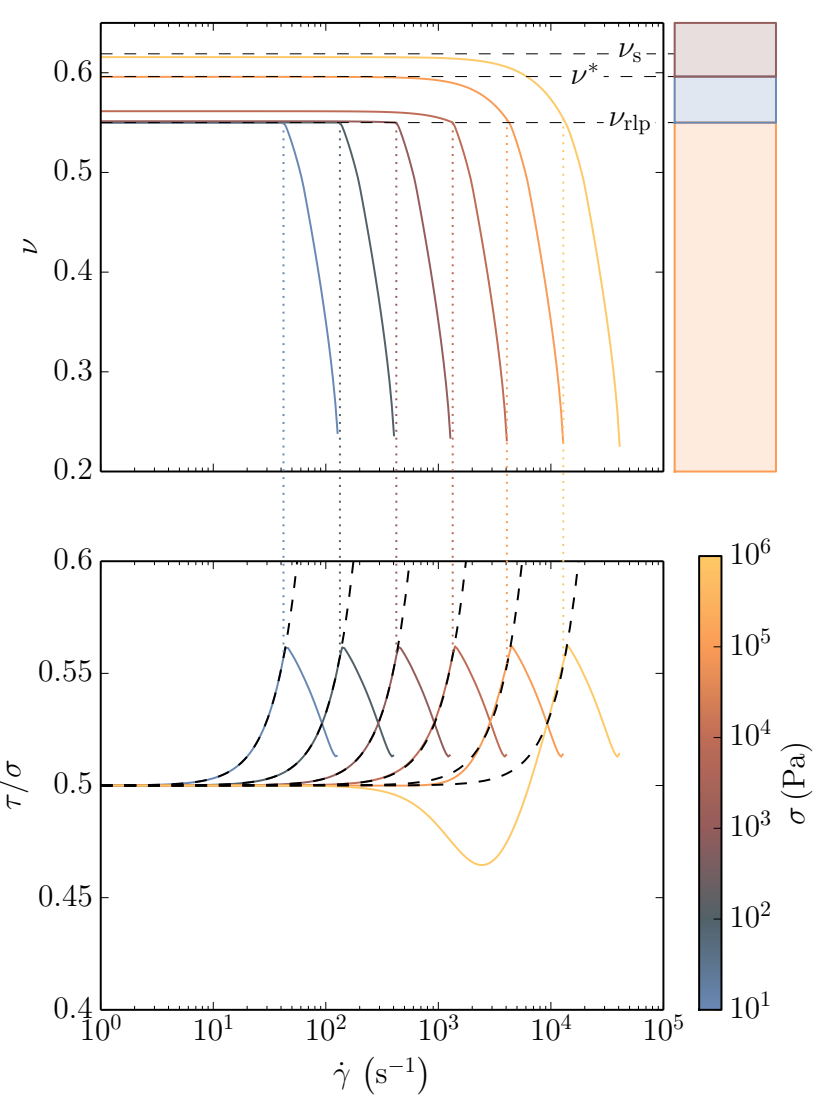

Figure 9. Concentration $v$ (top) and stress ratio $\tau / \sigma$ (bottom) as proposed by the extended kinetic theory (colored solid lines) in comparison to the simplified relation (black dashed lines in bottom figure) for the idealized quartz sand. The simplified relation for the stress ratio approximates the complex extended kinetic theory good for concentrations between $v_{\mathrm{rlp}}$ and $v^{*}$, e.g., in the monotonic dense flow regime (blue area, top-right). Between $v^{*}$ and $v_{\mathrm{S}}$ the regime is dense but non-monotonic (red area, top-right), below $v_{\text {rlp }}$ the regime is purely collisional (yellow area, top-right) and the concentration decreases rapidly with increasing shear rate.

small, as for the middle part of an avalanche (referred to as equilibrium shape of the velocity profile in Issler and Gauer, 2008, or simple shear infinite landslide model in Dutto, 2014), all volume forces and stresses can be expressed with the differential equations

$$
\begin{aligned}
& \frac{\partial \tau}{\partial z}=-\rho g \sin \alpha, \\
& \frac{\partial \sigma}{\partial z}=-\rho g \cos \alpha .
\end{aligned}
$$

The left-hand side in Eqs. (47) and (48) describes the change of stresses in $z$ direction, which is caused by the gravitational volume force (right-hand side). Introducing the constitutive relation (Eq. 45) in Eq. (47) leads to

$$
\frac{\partial}{\partial z}(\mu \sigma)+\frac{\partial}{\partial z}\left(\lambda \dot{\gamma}^{2}\right)=-\rho g \sin \alpha,
$$




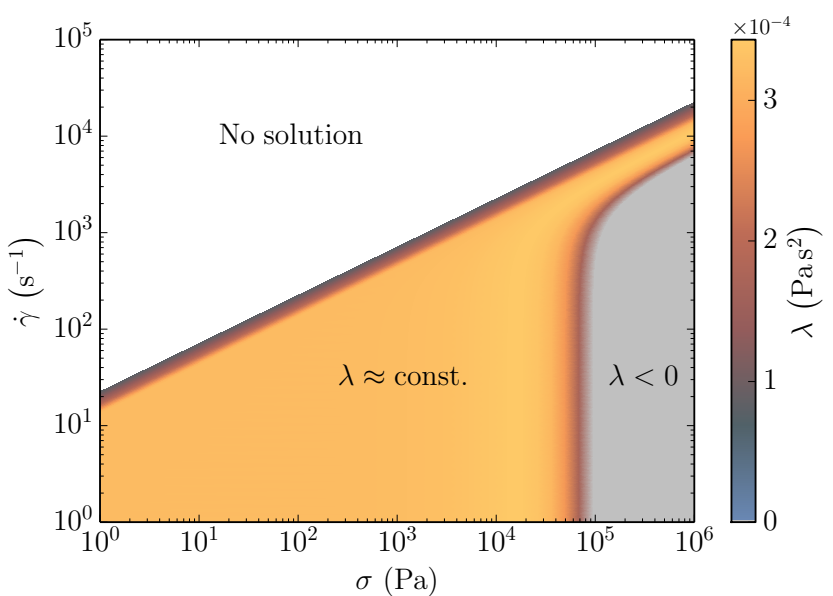

Figure 10. The factor $\lambda$ as a function of $\sigma$ and $\dot{\gamma}$. In the white area no solutions could be obtained with the model. In the yellow area the value for the factor $\lambda$ has its maximum value of approximately $3.5 \times 10^{-4} \mathrm{Pas}^{2}$. This value decreases at the borders of the yellow area. In the gray area, values for $\lambda$ are negative, indicating a nonmonotonic behavior.

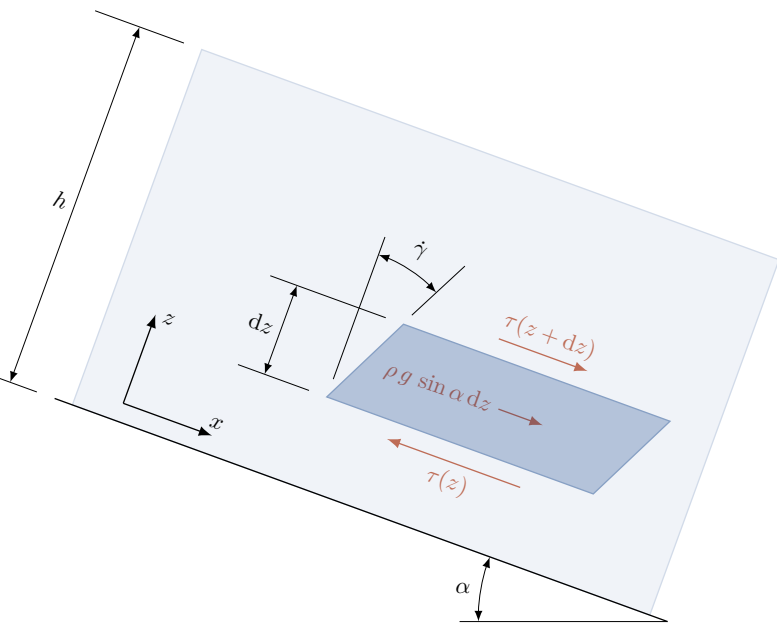

Figure 11. Orientation of the coordinate system and stresses in the slope parallel direction on an infinitesimal small control volume. Slope parallel normal stresses $\left(K_{\mathrm{a} / \mathrm{p}} \sigma\right)$ cancel each other and are not shown.

and with Eq. (48) for $\frac{\partial \sigma}{\partial z}$ to

$\frac{\partial}{\partial z}\left(\lambda \dot{\gamma}^{2}\right)=\rho g(\mu \cos \alpha-\sin \alpha)$.

Integration with respect to the boundary condition $\left.\dot{\gamma}\right|_{z=h}=0$ (following from $\left.\tau\right|_{z=h}=0$ ) leads to

$\dot{\gamma}=\sqrt{\frac{\rho g(\sin \alpha-\mu \cos \alpha)}{\lambda}} \sqrt{h-z}$.

Introducing $\dot{\gamma}=\frac{\partial u}{\partial z}$ and integrating again with respect to the boundary condition $\left.u\right|_{z=0}=0$ (no slip condition) leads to

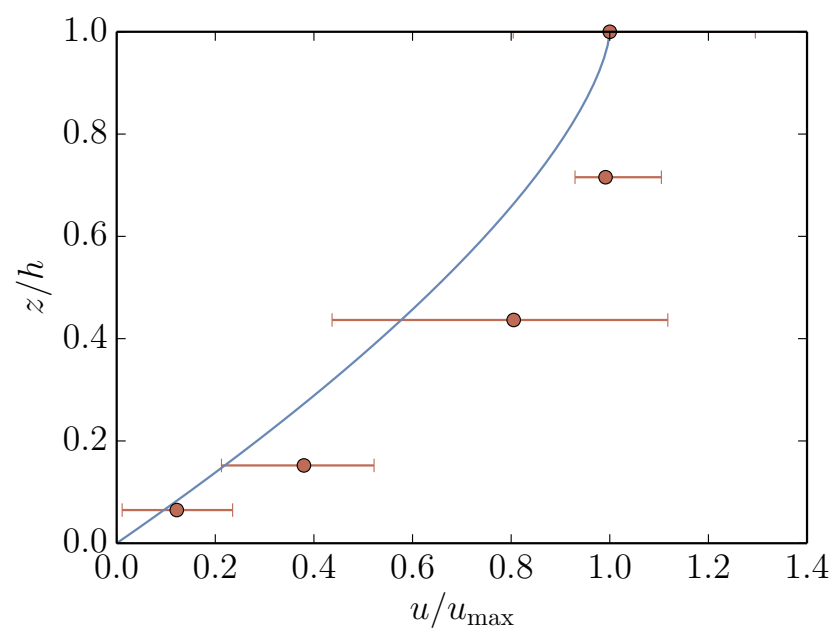

Figure 12. Velocity profile for an infinite avalanche in steady state on a uniformly steep slope for the given rheology model (blue line). For comparison, velocity measurements of a dry snow avalanche from a field test in Vallée de la Sionne is shown (red filled circle is the mean velocity; bars are fluctuations) (data from Kern et al., 2009; Sovilla et al., 2015). The measurement shows a more bulbous velocity profile, which indicates a plug flow regime. The bars show the high fluctuation of velocity of grains, which agrees with the assumptions of the kinetic theory.

an algebraic expression of the velocity profile, the so-called Bagnold profile (e.g., Pouliquen and Forterre, 2009):

$u=\frac{2}{3} \sqrt{\frac{\rho g(\sin \alpha-\mu \cos \alpha)}{\lambda}}\left(h^{3 / 2}-(h-z)^{3 / 2}\right)$.

The depth-averaged velocity can be calculated with

$\bar{u}=\frac{1}{h} \int_{0}^{h} u(z) \mathrm{d} z=\frac{2}{5} \sqrt{\frac{\rho g(\sin \alpha-\mu \cos \alpha)}{\lambda}} h^{3 / 2}$.

Molecular dynamic simulations of granular particles on an inclined plane result in a similar velocity profile, yielding an averaged velocity of $\bar{u} \propto h^{1.52 \pm 0.05}$ (Silbert et al., 2001). Moreover, this correlation was observed in experiments by Pouliquen (1999). Also, a comparison with velocity profile measurements in real-scale test sites (Kern et al., 2009; Sovilla et al., 2015) shows resemblance in the middle part of the avalanche; see Fig. 12.

The obtained velocity profile differs from the plug flow profile assumed by Savage and Hutter (1989). Consistently, the convective flux in the momentum Eq. (2) should be corrected with the shape factor (e.g., Baker et al., 2015). However, because of the small influence of this factor (Christen et al., 2010) and potential numerical problems (Hogg and Pritchard, 2004; Baker et al., 2015), the shape factor is set to 1 .

Finally a relation between the depth-averaged velocity (Eq. 53) and the shear rate at the bottom of the avalanche 
$\dot{\gamma}_{\mathrm{b}}=\left.\dot{\gamma}\right|_{z=0}($ Eq. 51) can be derived:

$\dot{\gamma}_{\mathrm{b}}=\frac{5}{2} \frac{\bar{u}}{h}$.

Introducing Eq. (54) into the constitutive relation leads to the basal shear stress

$\tau_{\mathrm{b}}=\mu \sigma_{\mathrm{b}}+\lambda\left(\frac{5}{2}\right)^{2}\left(\frac{\bar{u}}{h}\right)^{2}$.

The factor $5 / 2$ is directly related to the shape of the velocity profile and will change for other profiles, e.g., the velocity profile at the front of the avalanche. Moreover, a plug flow near the free surface is not reproduced by Eq. (55) but is visible in the measurement shown in Fig. 12. Dent et al. (1998) show velocity profiles where most shearing is concentrated at the ground. The appearance of a plug flow in measurements can be explained with cohesion (Norem et al., 1987), segregation effects or a flow in transitional state (see also Rauter, 2015, for velocity profiles in transitional states).

Expression (55) appears similar to classic friction relations. For example, the relation predicted by Issler and Gauer (2008), who split friction into a quasi-static and dynamic part. In their model, quasi-static stresses are reduced for low concentrations, referring to states with $v<v_{\text {rlp }}$ as fluidized. Instead of employing a microrheological model, such as kinetic theory, their relation is based on the macroscopic Norem-Irgens-Schieldrop rheology (Norem et al., 1987). Also the similarity to the Voellmy friction relation, given as

$\tau_{\mathrm{b}}=\mu \sigma_{\mathrm{b}}+\frac{\rho g}{\xi} \bar{u}^{2}$

is remarkable. Because of the similarity and its extensive application in snow avalanche simulations, the Voellmy friction relation is used as a reference to test the performance of the one derived here. For a better comparison to the Voellmy friction parameter $\xi$ the parameter

$\chi:=\left(\frac{2}{5}\right)^{2} \frac{\rho g}{\lambda}$

is introduced. This leads to the expression

$\tau_{\mathrm{b}}=\mu \sigma_{\mathrm{b}}+\frac{\rho g}{\chi} \frac{\bar{u}^{2}}{h^{2}}$,

where $\chi$ contains the velocity profile dependent factor $5 / 2$. A constant $\chi$ indicates that the same shape of the velocity profile in the whole avalanche is assumed.

The difference between the obtained relation and the friction relation of Voellmy is the inverse quadratic dependency on the flow height. This leads to a lower friction for larger flow heights and therefore larger avalanches. This behavior is in line with observations, where the runout is often related to the volume of the avalanche (e.g., Scheidegger, 1973; Bovis and Mears, 1976; Körner, 1980; Alean, 1985).

\section{Model test and parameter evaluation}

To test the obtained friction relation, we employ a multivariate optimization method, based on the work of Fischer et al. (2015). This method takes different optimization variables into account, which represent the main avalanche characteristics, e.g., runout or velocity. These can be obtained from simulations and field observations and their residuals can be quantitatively evaluated. Low residuals indicate a good simulation-observation correspondence. The variation of input parameters is limited to the friction parameters, which allows a simple and clear comparison. By scanning the entire physically relevant parameter space, parameter sets, yielding minimal residuals between simulation and observation, are identified. The combination of two different avalanche events, which differ significantly in volume and velocity, is investigated, allowing us to unify parameter sets for avalanches of different types, which is usually not only a superposition of the single events (compare Issler et al., 2005). The two avalanche events are (compare Fischer et al., 2014)

- avalanche no. 103 from 10 February 1999 at the Vallée de la Sionne (VdlS) test site with a deposition volume of approximately $500000 \mathrm{~m}^{3}$ and a velocity of up to $70 \mathrm{~ms}^{-1}$ (see Sovilla, 2004; Sovilla et al., 2006, for details) and

- avalanche from 17 April 1997 at the Ryggfonn (Rgf) test site with a deposition volume of approximately $40000 \mathrm{~m}^{3}$ and a velocity of up to $40 \mathrm{~m} \mathrm{~s}^{-1}$ (see Gauer et al., 2007, for details).

The simulations have been carried out using the SamosAT simulation software, including entrainment and the respective friction relation. To calculate the earth pressure coefficient $K_{\mathrm{a} / \mathrm{p}}$, a value of $15^{\circ}$ for the internal friction angle $\phi$ is used in all simulations. Note that in SamosAT, $\phi$ is set equal to $\delta$, when $\phi<\delta$, to solve Eq. (4).

Especially for the VdlS avalanche, entrainment appears important because of the high increase of volume during its descent. A simple approach for the entrainment rate $\dot{q}$ of the form

$\dot{q}=\frac{\tau_{\mathrm{b}}}{e_{\mathrm{b}}}|\bar{u}|$,

where $e_{\mathrm{b}}$ represents the specific erosion energy (compare Fischer et al., 2015) is employed. To estimate appropriate erosion energy coefficients we calculated growth indices, determining the quotient of the deposition mass and the initially released mass. This index is mainly influenced by the entrainment model, the available snow mass and the corresponding parameter. The field observations yield growth indices of 2.3 and 6.0 for Rgf and VdlS, respectively. To resemble values in this range, erosion energy coefficients of $10^{4} \mathrm{~J} \mathrm{~kg}^{-1}$ for Rgf and $10^{3} \mathrm{~J} \mathrm{~kg}^{-1}$ for VdlS were found to be appropriate. The 
snow cover height $h_{\mathrm{msc}}$, which is used to limit the entrainment and determine the release volume, was calculated with regards to the elevation and slope inclination $\alpha$ :

$h_{\mathrm{msc}}=h_{0}+\Delta h\left(z-z_{\mathrm{ref}}\right) \cos \alpha$,

where $h_{0}$ represents the snow cover height at the elevation $z_{\text {ref }}$ and $\Delta h$ represents its increase with elevation. This approach ensures a smooth initial snow distribution. The snow cover parameters $\left(z_{\text {ref }}=2400 \mathrm{~m}, h_{0}=1 \mathrm{~m}, \Delta h=10^{-4}\right.$ for VdlS and $z_{\text {ref }}=1500 \mathrm{~m}, h_{0}=2 \mathrm{~m}, \Delta h=6 \times 10^{-5}$ for Rgf) are chosen to match field observations of release volume and snow depth estimates.

In order to investigate the range of possible simulation results, the friction parameter $\mu$ is varied uniformly between 0.1 and 0.5 for both friction models, the friction parameters $\xi$ and $\chi$ are varied between $10^{2}$ and $10^{4}$ (units are $\mathrm{m} \mathrm{s}^{-2}$ and $\mathrm{m}^{-1} \mathrm{~s}^{-2}$, respectively). A logarithmic distribution for these parameters is chosen in order to account for the large associated uncertainty, i.e., order of magnitude.

To judge the quality of our simulations, they are compared with measurements of the following three observation variables.

The velocity in the avalanche track obtained by pulsed Doppler radar measurements. The radar measures the radial velocity and in combination with the elevation model, the surface parallel velocity can be calculated. After an appropriate coordinate transformation these values can then be compared with velocities obtained by simulations (Fischer et al., 2014). The radars settings allowed a distance between range gates of $50 \mathrm{~m}$. This leads to a resolution of 14 values along the avalanche path for both events.

The affected area near the deposition area. The deposition area cannot be analyzed directly because the dynamic model does not simulate the deposition process explicitly. Therefore areas where the simulation results exceed a specific dynamic peak pressure, $p_{\text {lim }}=1 \mathrm{kPa}$ in our case, are compared. The dynamic pressure is calculated from primary flow variables as

$p=\rho \bar{u}^{2}$,

with $\rho=200 \mathrm{~kg} \mathrm{~m}^{-3}$. Note that the simulation results are independent of the density $\rho$ and the pressure limit $p_{\text {lim may }}$ equivalently be expressed in terms of peak velocities. However, defining affected areas and runout in terms of pressure is in accordance with different international hazard mapping guidelines (c.f. Jóhannesson et al., 2009).

The runout distance along the avalanche path. The runout length is measured as projected length in the natural coordinate system, defined by the avalanche track. Just like the affected area, the runout length is defined by the farthest point where the avalanche exceeds the pressure $p_{\text {lim }}$ along its cross section (Fischer, 2013). To quantify the quality of a simulation with the parameter set $i$, we used the residuals between values obtained by the respective simulation $X_{i}$ and the measurements $\hat{X}$, calculated as

$\delta X_{i}=\left|X_{i}-\hat{X}\right|$,

where $\delta X$ can be the residual of velocity $\delta \bar{u}$ or of runout length $\delta r$. The residual of the affected area $\delta A$ is calculated in a similar manner but integrated over the investigated area $A_{\mathrm{oi}}$

$\delta A_{i}=\int_{A_{\mathrm{oi}}}\left|a_{i}(x, y)-\hat{a}(x, y)\right| \mathrm{d} A$.

Here, $a_{i}(x, y)$ denotes whether the pressure exceeded the threshold $p_{\lim }$ at the respective position $x, y$ in the simulation $i$ or not:

$a_{i}(x, y)=\left\{\begin{array}{ll}1 & \text { if } \quad p_{i}(x, y) \geq p_{\lim } \\ 0 & \text { otherwise }\end{array}\right.$.

$\hat{a}(x, y)$ represents the documented affected area in the same manner. Therefore, $\delta A_{i}$ represents the area where simulation and documentation disagree. In this way we could take into account not only the runout distance from a single point but also the shape of the avalanche. The area where the affected area was analyzed ( $A_{\mathrm{oi}}$, area of interest, in Eq. 63) is shown in Fig. 13. It contains the whole runout area of all simulations and the documented affected area. To combine residuals expressed by more than one value (like the velocity in the avalanche track, represented by a value for each range gate) we used a value related to the residual sum of squares of the form

$\delta X_{i}=\sqrt{\frac{\sum_{n=1}^{N} \delta X_{i, n}^{2}}{N}}$.

The division by the number of values $N$ and taking the square root ensures that the resulting residual is of the same unit and of comparable size with respect to the single values. This eases the interpretation from an engineering point of view. We used the same concept to combine residuals from more events to obtain a single residual which would be obtained by simulating these events with the same parameters. The events in this paper are VdlS and Rgf:

$\delta X_{i, \mathrm{Vdl}+\mathrm{Sgf}}=\sqrt{\frac{\delta X_{i, \mathrm{VdlS}}^{2}+\delta X_{i, \mathrm{Rgf}}^{2}}{2}}$.

To combine residuals of different kinds, like runout and velocity, we normalized the respective residuals with the minimum and maximum residuals from all simulations to eliminate the specific scale:

$\delta X_{i, \text { norm }}=\frac{\delta X_{i}-\delta X_{\min }}{\delta X_{\max }-\delta X_{\min }}$, 
$\delta X_{i, \mathrm{comb}}=\sqrt{\frac{\delta \bar{u}_{i, \mathrm{norm}}^{2}+\delta r_{i, \mathrm{norm}}^{2}+\delta A_{i, \mathrm{norm}}^{2}}{3} .}$

The normalization was always performed after the combination of values of the same kind and after combining two events. This is important because the normalization and combination of residuals is not commutative.

This method does not require reference values like an acceptable error or a measurement error. A possible drawback is that larger events have a bigger impact on the results than smaller ones because of the larger absolute values of velocity and runout. If this is not suitable for the respective problem, one could also perform the normalization before combining the events and therefore lay weight on different events equally. The combination of events and measures leads to four possibilities to evaluate and compare model performance with respect to different regards and events (compare Figs. 15, 16 and Table 2):

a. to a single event with respect to a single observation variable $(\delta r, \delta A$ and $\delta \bar{u}$, marked by $\Delta, \Delta, \Delta)$

b. to a single event with respect to all investigated observation variables $(\delta r \wedge \delta A \wedge \delta \bar{u}$, marked by $\nabla)$

c. to both events with respect to a single observation variable $\left(\delta r_{\mathrm{VdlS}+\mathrm{Rgf}}, \delta A_{\mathrm{VdlS}+\mathrm{Rgf}}\right.$ and $\delta \bar{u}_{\mathrm{VdlS}+\mathrm{Rgf}}$, marked by $\circ, \circ, \bigcirc)$

d. to both events with respect to all investigated observation variables $\left(\delta r_{\mathrm{Vdl} I S+\mathrm{Rgf}} \wedge \delta A_{\mathrm{Vdl} S+\mathrm{Rgf}} \wedge \delta \bar{u}_{\mathrm{VdlS}+\mathrm{Rgf}}\right.$, marked by $\circ$ )

The first evaluation is the simplest to be fulfilled sufficiently with the simulation results. The last contains the most information and is therefore the most valuable.

\section{Results and Discussion}

\subsection{Simulation results}

The following section shows the evaluation of 1600 simulation runs. This number results from two events, two friction models and 20 values for the friction parameters $\mu$ and $\xi$ or $\chi$, respectively.

In Fig. 15 the evaluation of residuals from all simulations is summarized, separated by event and friction model. Figure 16 shows the same result combined for both events. Additionally the combined residuals in dependence of the respective friction parameters are highlighted.

\subsection{Predictive power of employed friction relations}

To gain a first insight concerning the validity of the backcalculated model parameters for the two investigated friction relations, we determine the optimal parameter set for the Rgf event and obtain $\mu=0.437, \xi=10000 \mathrm{~m} \mathrm{~s}^{-2}$ for the Voellmy relation and $\mu=0.395, \chi=7848 \mathrm{~m}^{-1} \mathrm{~s}^{-2}$ for the kinetic theory relation. Prediction of the VdlS event with these parameters yields a global residual of $0.27\left(\delta r=300 \mathrm{~m}, \delta A=140000 \mathrm{~m}^{2}, \delta \bar{u}=18 \mathrm{~m} \mathrm{~s}^{-1}\right)$ for the Voellmy relation and $0.15\left(\delta r=180 \mathrm{~m}, \delta A=98000 \mathrm{~m}^{2}\right.$, $\delta \bar{u}=13 \mathrm{~m} \mathrm{~s}^{-1}$ ) for the kinetic theory relation. Switching the events and performing the same procedure (i.e., utilizing the parameters optimized for the VdlS event for a Rgf prediction) leads to a global residual of $0.26(\delta r=120 \mathrm{~m}$, $\delta A=35000 \mathrm{~m}^{2}, \delta \bar{u}=9 \mathrm{~m} \mathrm{~s}^{-1}$ ) for the Voellmy relation and $0.19\left(\delta r=110 \mathrm{~m}, \delta A=24000 \mathrm{~m}^{2}, \delta \bar{u}=9 \mathrm{~m} \mathrm{~s}^{-1}\right)$ for the kinetic theory relation. These residuals indicate an enhanced prediction with the presented friction relation in comparison to the Voellmy reference for both cases.

\subsection{Single and combined parameter optimization}

The runout distance represents a point in the avalanche path. Simulations with high friction (high values for $\mu$, low values for $\xi$ and $\chi$ ) may not reach this point, simulations with low friction may exceed this point. Between those limiting cases, a simulation with optimal parameters fits the documented runout almost perfectly. In order to satisfy two observations from two events, each simulation demands its own optimal parameter set (Fig. 15). Therefore, simulations with the same parameter set for two events will lead to a residual between predicted and documented runout (Fig. 16). The residual for the runout length is $22.9 \mathrm{~m}$ for the kinetic theory and $27.4 \mathrm{~m}$ for the Voellmy relation at best when optimizing both events together.

The affected area is also a measure related to runout. However, it provides an additional important information on the lateral extend and spatial distribution of the avalanche. The correlation to runout is clearly visible in Figs. 15 and 16, as the respective areas of low residuals overlap. Figure 13 shows the documented affected area alongside with the affected area obtained from simulations with the smallest residuals in this respect $\left(\delta A_{\min }\right)$. A perfect correspondence between the documented area and the affected area in the simulation does not appear in any of our simulation runs. This can also be seen in Fig. 15. The smallest residual is about $7700 \mathrm{~m}^{2}$ for Rgf and $65000 \mathrm{~m}^{2}$ for VdlS.

In case of the Rgf avalanche, it is observed that the agreement of documented and simulated affected area is limited. This can be attributed to a large amount of deposited snow in the runout, which is not considered in the digital elevation model, leading to an upstream spreading of the avalanche (see Fig. 13a). All simulations are affected equally by this effect, which leads to the big red areas in Fig. 15a and b.

For the VdlS event, the delineation of the documented affected area is accompanied by high uncertainties due to the large powder cloud of this avalanche. The applied documented affected area represents areas with clearly visible snow depth variations (deposition and erosion) caused by the avalanche (Vallet et al., 2001). Figure 13b shows that simula- 


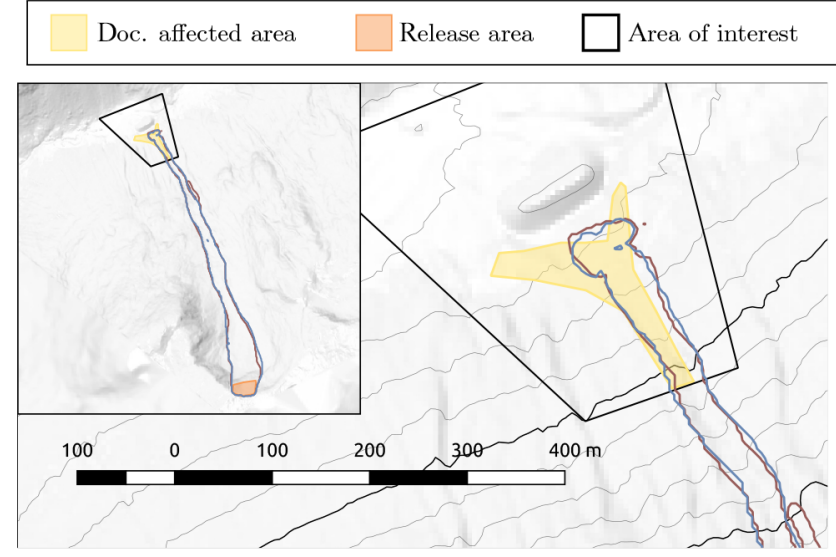

(a) Ryggfonn

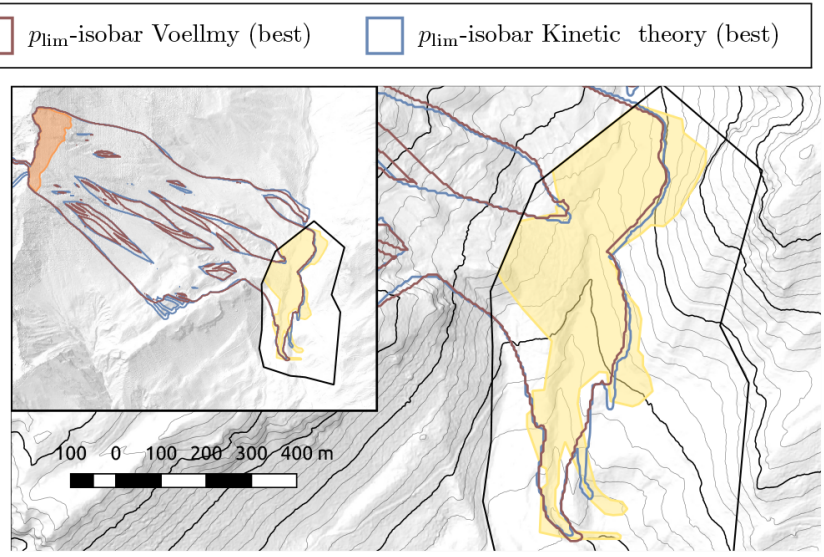

(b) Vallée de la Sionne

Figure 13. Outlines of the numerical simulations in comparison with the documented affected area for the avalanche event in Ryggfonn (a) and Vallée de la Sionne (b). The red and blue lines show the $p_{\text {lim }}$ isobars for the simulation with the smallest residuals in affected area $\left(\delta A_{\min }\right)$ for the Voellmy friction model and the new friction model, respectively. The yellow filled areas show the documented affected areas and the orange filled areas show the release areas. The evaluation of the affected area was limited to the area within the black polygons. In the figure showing the event in Rgf one can see that the avalanche (yellow area) stopped and spread apart about $50 \mathrm{~m}$ before the dam. This results from a large snow deposit uphill of the dam. Because the digital elevation model does not take into account the snow height, numerical simulations show the same behavior of the avalanche with an offset of about $50 \mathrm{~m}$. The smallest residual is achieved by simulations with high friction, which leads to a stopping of the avalanche before reaching the dam and an untypical form.

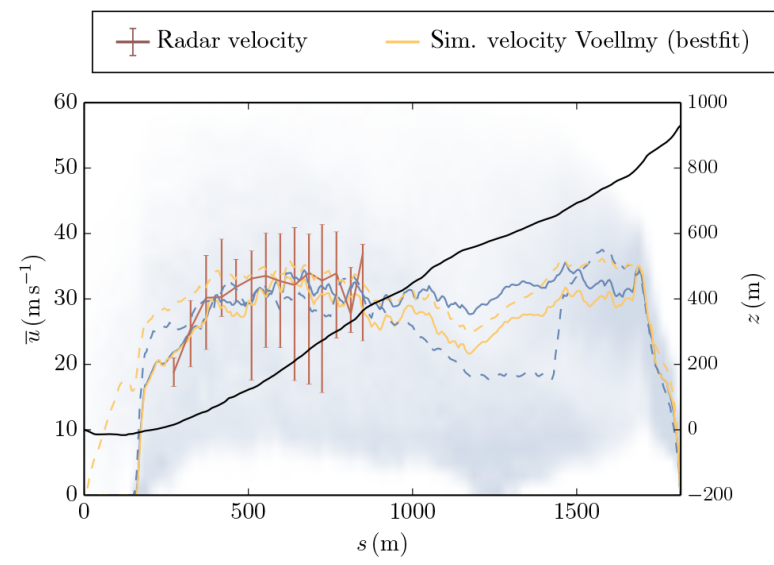

(a) Ryggfonn

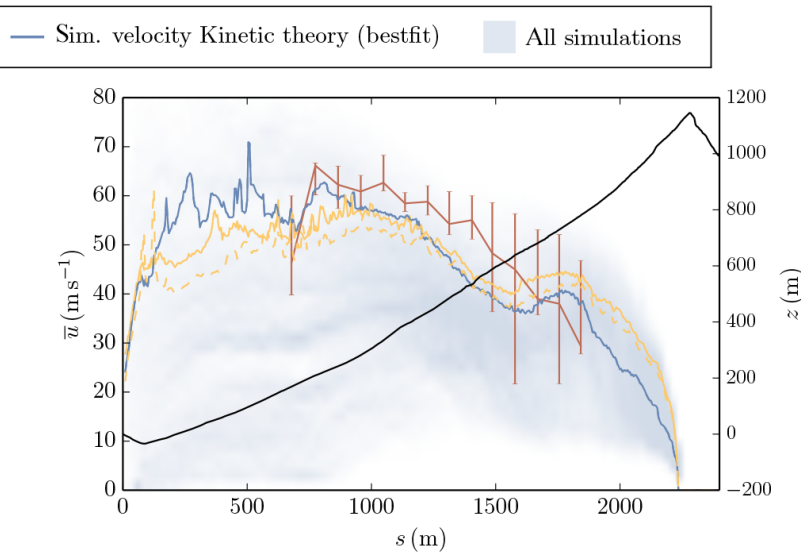

(b) Vallée de la Sionne

Figure 14. Velocity measurements compared with simulation outcomes for the avalanche event in Ryggfonn (a) and Vallée de la Sionne (b). The flow direction is right to left. The $x$ axis shows the distance from the radar station and the left $y$ axis shows the velocity. For a better orientation the elevation profile is shown in black and the right $y$ axis shows the elevation. The red line shows the velocity obtained by the pulsed Doppler radar measurements with an estimated observational error. The yellow and blue lines show the velocity along the radar path in the simulation with the smallest residual for the Voellmy friction model and the new friction model, respectively. The dashed lines show the best simulation when using the same material parameter for both events. For the kinetic theory friction model the optimized parameter set for VdlS and both events combined coincide. The background shows the distribution of velocities obtained by all simulations.

tions with an overall good accordance in the runout fail to reproduce the high climb on the counter slope (the two humps opposite to the two main avalanche tracks). This is the exact area where one expects the powder snow layer to detach from the dense flow layer (the dense flow layer follows the terrain more strictly than the powder snow layer).
Another interesting detail can be observed in Fig. 13b. In contradiction to the Voellmy relation, the kinetic theory relation predicts a separation of two branches in the runout zone, which matches the observed behavior. This is an indication for a proper description of important physical processes in the avalanche. 
The velocity along the radar path is visualized in Fig. 14 . The residuals are shown in Fig. 15. For the Rgf event the smallest residual among all simulations is $2.4 \mathrm{~m} \mathrm{~s}^{-1}$ and for the event in the VdlS the smallest residual is $6.2 \mathrm{~m} \mathrm{~s}^{-1}$.

In Fig. 14a the dashed yellow line, representing the Voellmy simulation with the smallest residual in velocity for both events combined, matches the observed velocity quite well. However, the runout prediction of the respective simulation appears to be inaccurate. This example shows the importance of the evaluation of different observation variables.

The dynamic pressure can be calculated from velocity with Eq. (61). The residual follows from Eq. (62) as for other observation variables. A direct calculation of the residual in pressure using the residual in velocity is not possible because of their nonlinear correlation. Minimal residuals in velocity yield minimal residuals in pressure of 28 and $118 \mathrm{kPa}$ for $\mathrm{Rgf}$ and VdlS, respectively. Minimal residuals differ only slightly between the two investigated friction relations, when optimizing on single events. However, when using the same set of material parameters for both events, the residual gained with the kinetic theory model is $88 \mathrm{kPa}$ compared to $116 \mathrm{kPa}$ from calculation with the Voellmy model (about $25 \%$ ).

\subsection{Combinations and parameter matching}

Possible best-fit parameters can be obtained by analyzing the overlapping areas in Fig. 15. This areas represent parameter combinations which yield relatively small residuals. Figure 16a and $b$ show the same kind of areas for a combination of the two events. The bigger influence of the event in VdlS is clearly visible, as Fig. 16a and b are quite similar to Fig. 15c and d. The white circles in Fig. 16 mark the positions of the smallest combined residual. In both cases it is located in the overlapping area of relatively small individual residuals.

The combined residual of velocity, runout length and affected area is shown in Fig. 16c and d for both friction relations. The position of the minimal combined residuals in the parameter space is marked with white circles. The form of the isolines in Fig. 16c and d matches qualitatively the overlapping areas of small residuals in Fig. 16a and b. The combined normalized residual as calculated here seems an appropriate method for the determination of optimized parameter sets.

The parameters for the kinetic theory model, which yield the smallest combined residual for both events (e.g., residual in velocity, runout and affected area, white circles in Fig. $16 \mathrm{~b}$ and d), are $\mu=0.311, \chi=3793 \mathrm{~m}^{-1} \mathrm{~s}^{-2}$. For the Voellmy model, these parameters are $\mu=0.268, \xi=$ $2976 \mathrm{~m} \mathrm{~s}^{-2}$ (white circles in Fig. 16a and c).

For comparison, practical guidelines propose the variation of Voellmy friction parameters with different avalanche characteristics; e.g., $\mu$ is varied between 0.155 for big avalanches and 0.3 for small avalanches (e.g., Salm et al., 1990; Gruber et al., 1999). This tendency is in accordance to the presented results $(\mu=0.437$ for $\mathrm{Rgf}$ and $\mu=0.268$ for VdlS). How- ever, values for $\mu$ found in this work are higher compared to the ones utilized in practice. For the turbulent friction parameter $\xi$, guidelines propose a relation to terrain features and suggest values between $500 \mathrm{~m} \mathrm{~s}^{-2}$ (high roughness, channeled avalanche path) and $1000 \mathrm{~m} \mathrm{~s}^{-2}$ (small roughness). It appears that the newly obtained values for the turbulent friction coefficient $\xi$ are about 1 magnitude larger, which is in accordance to previous observations (Fischer et al., 2015, and references therein). However a direct comparison between single model parameters is limited, since different model implementations (e.g., entrainment) lead to different optimal parameter settings.

\subsection{Back calculation of microscopic material parameters}

Since a direct measurement is hardly possible, optimized values for the parameters $\mu$ and $\chi$ can be combined with observable properties to estimate microscopic parameters of the extended kinetic theory in the spirit of Salm (1993).

The tangent of the critical friction angle $\tan \phi_{\mathrm{ss}}^{\prime}$ is equal to the Coulomb friction coefficient $\mu$. Moreover, $\tan \phi_{\mathrm{ss}}^{\prime}$ can be used to estimate limit values for the concentration $\nu$. The correlation between $\tan \phi_{\mathrm{ss}}^{\prime}$ and $v_{\mathrm{s}}$ has been investigated by Chialvo et al. (2012) using 3D DEM simulations. For $\tan \phi_{\mathrm{sS}}^{\prime}=0.405$, matching approximately the value obtained from the real-scale avalanche simulations (Rgf), Chialvo et al. (2012) obtain $v_{\mathrm{s}}=0.581$. With a similar approach, Silbert (2010) obtains $v_{\text {rlp }}=0.556$ for $\tan \phi_{\mathrm{ss}}^{\prime}=0.405$. The particle density $\rho_{\mathrm{p}}$ is assumed to be $300 \mathrm{~kg} \mathrm{~m}^{-3}$, following field measurements by McClung and Schaerer (1985) and experiments by Steinkogler et al. (2015a). This leads to a maximal bulk density of $174.3 \mathrm{~kg} \mathrm{~m}^{-3}$, which is reasonable for snow avalanches (e.g., Gauer et al., 2008) and close to the assumed value of $200 \mathrm{~kg} \mathrm{~m}^{-3}$ employed in this work to estimate the dynamic pressure. The Young's modulus $E$ is estimated using the empirical relation of Scapozza (2004),

$E=1.873 \times 10^{5} e^{0.0149 \rho_{\mathrm{p}}}$

where $\rho_{\mathrm{p}}$ is the particle density with unit $\mathrm{kg} \mathrm{m}^{-3}$ and $E$ has the unit Pa. For $\rho_{\mathrm{p}}=300 \mathrm{~kg} \mathrm{~m}^{-3}, E=1.64 \times 10^{7} \mathrm{~Pa}$ and $K=\pi d E / 8=1.29 \times 10^{5} \mathrm{Pam}$. The coefficient of restitution $\epsilon$ is assumed to be 0.1 , following Steinkogler et al. (2015a). The sensitivity on the parameter $c$ is rather small and the value of 0.5 is not changed. The macroscopic friction parameter $\lambda$ is calculated from $\chi=7848 \mathrm{~m}^{-1} \mathrm{~s}^{-2}$ (Rgf), using the correlation given by the velocity profile, Eq. (57), leading to $\lambda=0.04 \mathrm{~Pa} \mathrm{~s}^{2}$. The unknown microscopical material parameters $d$ and $a$ can be estimated by fitting Eq. (44) to $\lambda=0.04 \mathrm{~Pa} \mathrm{~s}^{2}$ for a specific range of $\sigma=\left[10^{2} \mathrm{~Pa} ; 10^{5} \mathrm{~Pa}\right]$ and $\dot{\gamma}=\left[10^{0} \mathrm{~s}^{-1} ; 10^{4} \mathrm{~s}^{-1}\right]$ or until $v<v_{\mathrm{rlp}}$. This leads to a particle diameter $d=0.02 \mathrm{~m}$ and a dimensionless coefficient for the critical state line $a=0.2$. The simplified friction relation with the respective macroscopic parameters obtained from the best Rgf simulation and the fitted ex- 


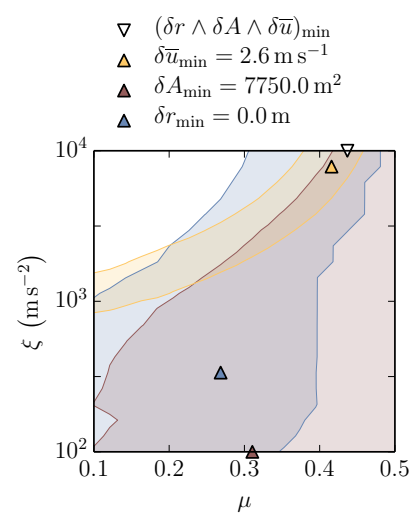

(a) Rgf/Voellmy

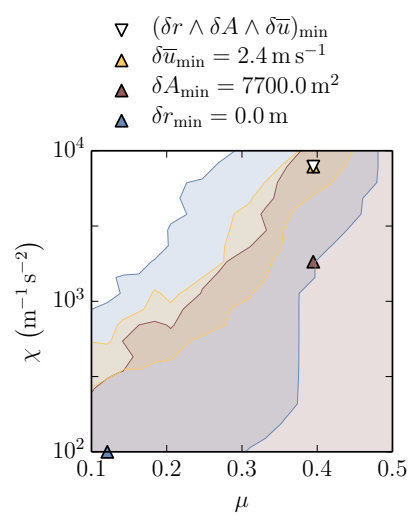

(b) Rgf/Kinetic theory

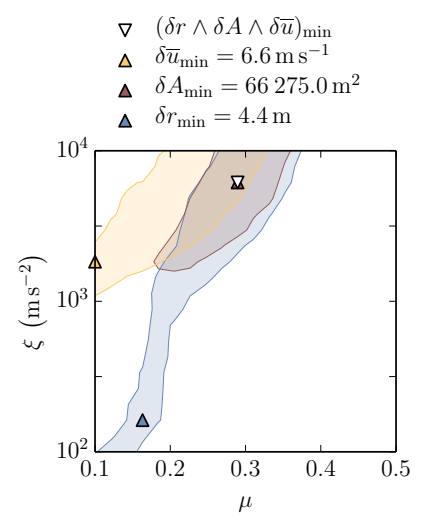

(c) VdlS/Voellmy

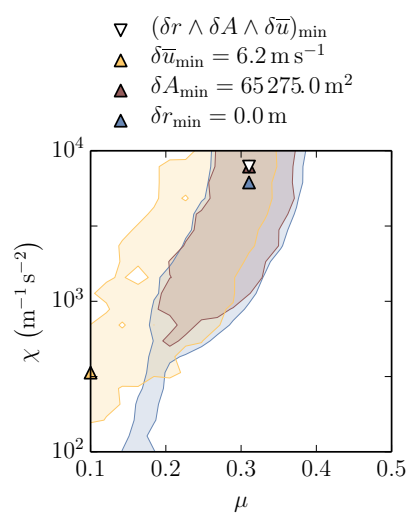

(d) VdlS/Kinetic theory

Figure 15. Areas in the parameter space with relatively small residuals (less than $10 \%$ on the normalized scale) for the runout length (blue), the affected area (red) and the velocity (yellow) for the different events and friction models. The triangle in the respective color marks the simulation with the smallest residual $\delta X_{\min }$. These residuals correspond to the evaluations of a single variable of a single event (type a). The white triangle marks the smallest combined residual, which corresponds to an evaluation of combined residuals of a single event (type b).

Table 2. Obtained residuals for all possible result evaluations. Each symbol marks a set of parameters which can also be seen in the parameter space in Figs. 15 and 16.

\begin{tabular}{|c|c|c|c|c|c|c|c|c|c|c|}
\hline \multirow[t]{2}{*}{ Method } & \multirow[t]{2}{*}{ Event } & \multicolumn{3}{|c|}{ Voellmy } & \multicolumn{3}{|c|}{ Kinetic theory } & \multicolumn{3}{|c|}{ Difference } \\
\hline & & $\delta r$ & $\delta A$ & $\delta \bar{u}$ & $\delta r$ & $\delta A$ & $\delta \bar{u}$ & $\delta r$ & $\delta A$ & $\delta \bar{u}$ \\
\hline (a) & $\operatorname{Rgf}$ & $\triangle 0.0 \mathrm{~m}$ & $\Delta 7750 \mathrm{~m}^{2}$ & $\Delta 2.6 \mathrm{~ms}^{-1}$ & $\triangle 0.0 \mathrm{~m}$ & $\Delta 7700 \mathrm{~m}^{2}$ & $\Delta 2.4 \mathrm{~m} \mathrm{~s}^{-1}$ & $0 \%$ & $1 \%$ & $9 \%$ \\
\hline (a) & VdlS & $\triangle 4.4 \mathrm{~m}$ & $\Delta 66275 \mathrm{~m}^{2}$ & $\triangle 6.6 \mathrm{~ms}^{-1}$ & $\triangle 0.0 \mathrm{~m}$ & $\Delta 65275 \mathrm{~m}^{2}$ & $\triangle 6.2 \mathrm{~m} \mathrm{~s}^{-1}$ & $100 \%$ & $2 \%$ & $5 \%$ \\
\hline (b) & $\operatorname{Rgf}$ & $\nabla 6.4 \mathrm{~m}$ & $\nabla 11675 \mathrm{~m}^{2}$ & $\nabla 2.9 \mathrm{~ms}^{-1}$ & $\nabla 12.9 \mathrm{~m}$ & $\nabla 10675 \mathrm{~m}^{2}$ & $\nabla 2.4 \mathrm{~m} \mathrm{~s}^{-1}$ & $-100 \%$ & $9 \%$ & $20 \%$ \\
\hline (b) & Vdls & $\nabla 13.3 \mathrm{~m}$ & $\nabla 66275 \mathrm{~m}^{2}$ & $\nabla 9.3 \mathrm{~ms}^{-1}$ & $\nabla 4.4 \mathrm{~m}$ & $\nabla 65275 \mathrm{~m}^{2}$ & $\nabla 8.5 \mathrm{~m} \mathrm{~s}^{-1}$ & $67 \%$ & $2 \%$ & $8 \%$ \\
\hline (c) & $\mathrm{Rgf}+\mathrm{VdlS}$ & ○ $27.4 \mathrm{~m}$ & - $53038 \mathrm{~m}^{2}$ & $6.2 \mathrm{~ms}^{-1}$ & - $22.9 \mathrm{~m}$ & - $49220 \mathrm{~m}^{2}$ & $5.0 \mathrm{~m} \mathrm{~s}^{-1}$ & $16 \%$ & $7 \%$ & $19 \%$ \\
\hline (d) & $\mathrm{Rgf}+\mathrm{VdlS}$ & $64.1 \mathrm{~m}$ & $\circ 57779 \mathrm{~m}^{2}$ & $\circ 8.1 \mathrm{~ms}^{-1}$ & $\circ 24.0 \mathrm{~m}$ & $\circ 49517 \mathrm{~m}^{2}$ & $\circ 7.0 \mathrm{~m} \mathrm{~s}^{-1}$ & $63 \%$ & $14 \%$ & $13 \%$ \\
\hline
\end{tabular}

tended kinetic theory relation are shown in Fig. 17. The full and simplified relation are in agreement for the dense flow regime, i.e., $v>v_{\text {rlp. }}$. At $v=v_{\text {rlp }}$ the stress ratio shows a sharp bend and the friction is overestimated by the simplified relation for $v<v_{\text {rlp }}$. The value for $\lambda$, obtained from the real-scale simulations, cannot be reconstructed with any particle diameter significantly higher then $0.02 \mathrm{~m}$ because $\lambda$ reacts much more sensitively to changes of $d$ than to any other parameter modification. Particle diameters in the deposition of avalanches vary between $0.035 \mathrm{~m}$ (smaller particle aggregates) and $0.335 \mathrm{~m}$ (large snow boulders) (Bartelt and McArdell, 2009). Steinkogler et al. (2015a) obtain diameters between $10^{-3} \mathrm{~m}\left(T<-1{ }^{\circ} \mathrm{C}\right), 0.02-0.1 \mathrm{~m}\left(-1^{\circ} \mathrm{C}<\right.$ $\left.T<0{ }^{\circ} \mathrm{C}\right)$ and $0.1-0.25 \mathrm{~m}\left(T>0{ }^{\circ} \mathrm{C}\right)$ in DEM simulations and laboratory experiments. It appears reasonable that the particle diameter significantly varies in flow and deposition and increases during descent, since the temperature is rising (Steinkogler et al., 2015b; Vera Valero et al., 2015). Consequently, one expects to find larger particles in the deposition area as in the flowing avalanche. In snow chute experiments Rognon et al. (2008) mostly observed particle diameters on a centimeter scale following a power law and Tiefenbacher and
Kern (2004) used a sub-centimeter setup to resolve snow particle and aggregate motion. Considering these experimental observations and earlier theoretical assumptions (e.g., Salm, 1993), a particle diameter of $0.02 \mathrm{~m}$ seems reasonable during avalanche motion.

\section{Conclusions}

From Fig. 15, one can see that both rheological models can be fitted almost equally well to single observations from field tests. The smallest residuals differ only slightly for the single cases. When different observation variables are combined, the kinetic theory approach allows a better fit to the observed data. This is indicated by the larger overlapping area of the three relatively small residuals in Fig. 16a compared to the areas in Fig. 16b. It stands to reason that the modification of the friction with the flow height can help to represent different stages or flow regimes of the avalanche better. This leads to a more realistic dynamic description in different parts of the avalanche, namely the avalanche track and the runout area. 


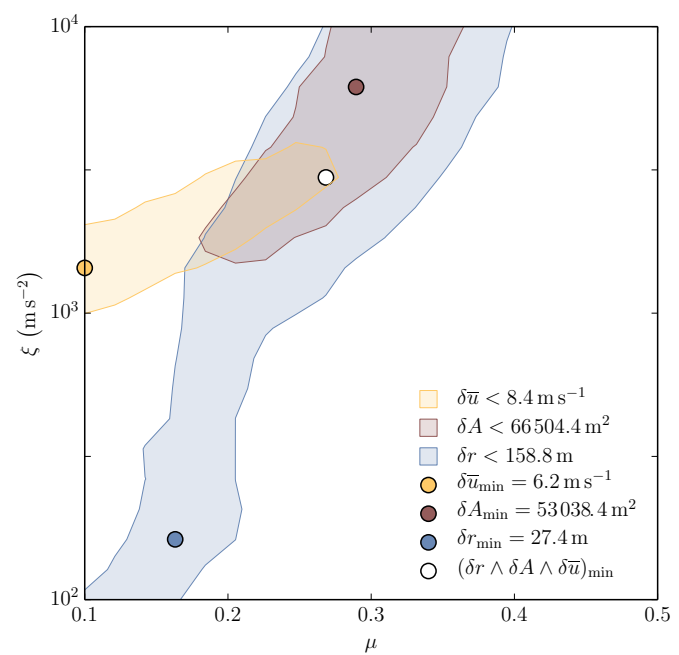

(a) Both events/Voellmy

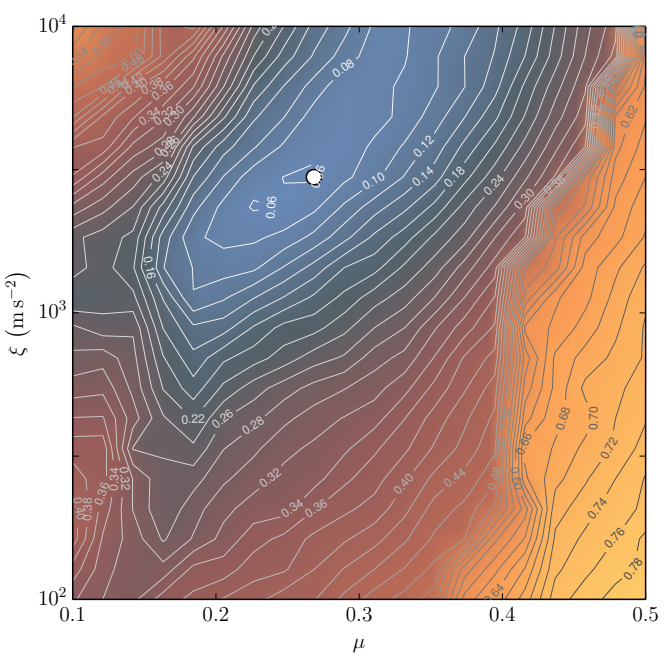

(c) Both events/Voellmy

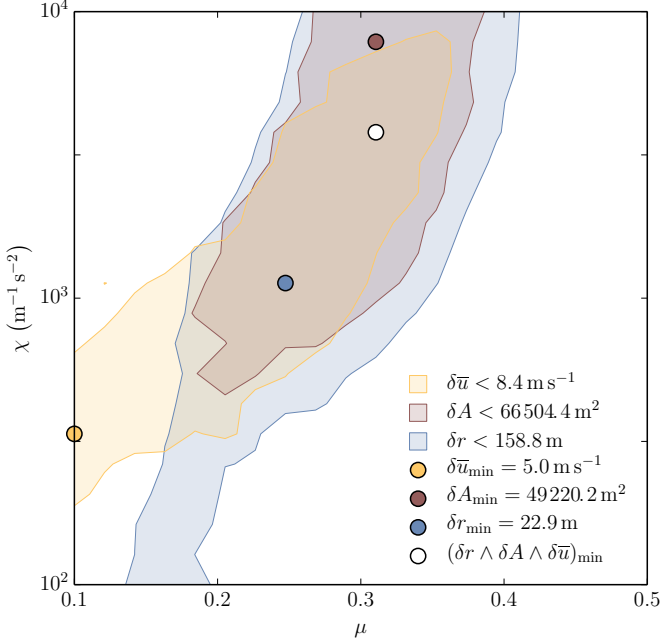

(b) Both events/Kinetic theory

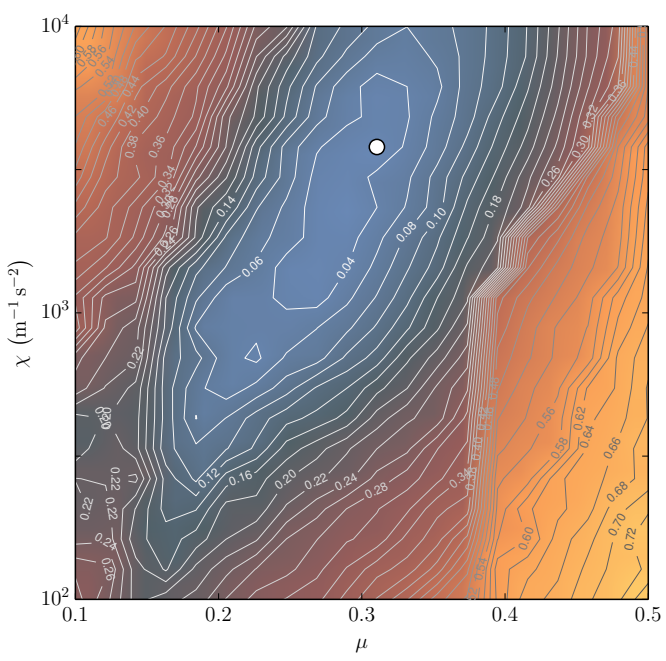

(d) Both event/Kinetic theory

Figure 16. (a, b) Areas in the parameter space with relatively small residuals (less than $10 \%$ on the normalized scale) for the runout length (blue), the affected area (red) and the velocity (yellow) for both events combined. The circle in the respective color marks the simulation with the smallest residual $\delta X_{\min }$ (optimization to a single variable for both events, type c). The white circle shows the minimal combined residual of runout, affected area and velocity (optimization, type d). (c, d) The combined residual in the parameter space. This surface has a clearly visible local minimum within the physical relevant area. Their position matches with the white circles in the graphs above. The minimal residual is in both cases located within the intersecting areas.

This tendency increases with the number of observations combined. Table 2 shows an overview over possible evaluations and values obtained for both investigated models, where this trend is clearly visible. The difference between the Voellmy relation and the kinetic theory approach increases with the number of combinations in the optimization process. Figure 16 shows residuals of the combination of events. The smallest combined residual for simulations with the Voellmy relation is 0.057 (combination of $\delta r=64 \mathrm{~m}$, $\delta A=58000 \mathrm{~m}^{2}, \delta \bar{u}=8 \mathrm{~m} \mathrm{~s}^{-1}$ and $\delta p=159 \mathrm{kPa}$ ). The kinetic theory approach reduces this value to 0.020 (combination of $\delta r=24 \mathrm{~m}, \delta A=50000 \mathrm{~m}^{2}, \delta \bar{u}=7 \mathrm{~m} \mathrm{~s}^{-1}$ and $\delta p=$
$132 \mathrm{kPa}$ ). This corresponds to a reduction of the residual in runout by about $60 \%$, alongside with a reduction of the residual in the pressure along the avalanche track by about $20 \%$.

This improvement can be obtained with very little modification to current models and simulation tools; i.e., no additional transport equation needs to be solved. Convection and diffusion are neglected and a local equilibrium of the granular temperature is assumed to get an analytical expression for the shear stress. An additional improvement with a more accurate description of the velocity profile is expected. A more realistic velocity profile should also lead to different friction 


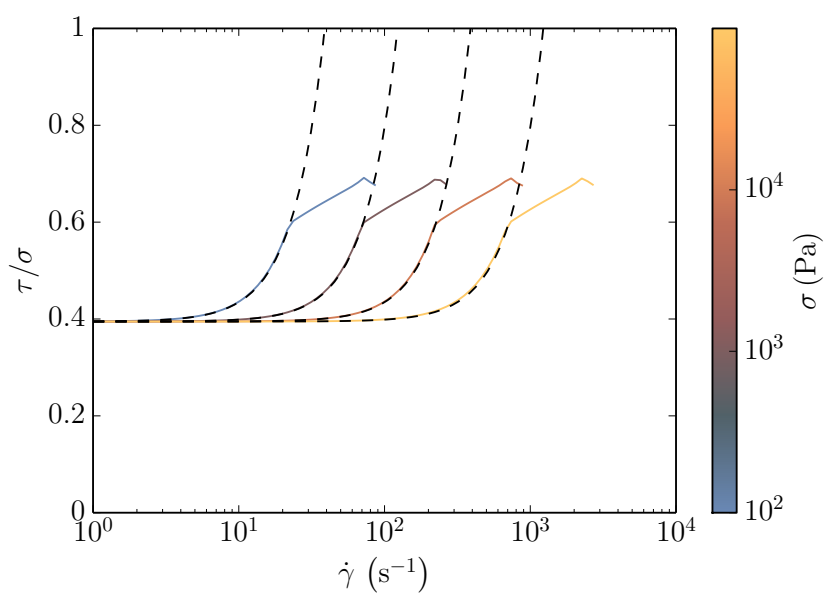

Figure 17. Stress ratio $\tau / \sigma$ according to the extended kinetic theory model (colored solid lines). Material parameters, $v_{\mathrm{rlp}}=0.556, v_{\mathrm{s}}=$ $0.581, \tan \phi_{\mathrm{ss}}^{\prime}=0.395, \rho_{\mathrm{p}}=300 \mathrm{~kg} \mathrm{~m}^{-3}, K=1.29 \times 10^{5} \mathrm{Pam}, \epsilon=$ $0.1, c=0.5, d=0.02 \mathrm{~m}, a=0.2$, are chosen to match the simplified friction model (black, dashed lines) with parameters $\mu=0.395$, $\lambda=0.04 \mathrm{Pas}^{2}, \chi=7848 \mathrm{~m}^{-1} \mathrm{~s}^{-2}$, respectively.

in head and tail of the avalanche like proposed by Buser and Bartelt (2009).

Overall, velocities predicted by the presented models can match the observations quite well with an optimized set of parameters. However, this may also be attributed to the considered entrainment process since the analysis of similar friction approaches showed less agreement of the velocities, disregarding entrainment (Fischer et al., 2014). This highlights the importance of considering friction and entrainment equally in a process-orientated approach and the respective impact on avalanche velocities along the track.

The evaluation of the affected area is accompanied by large uncertainties in the documentations. Therefore, assumptions about the quality of the model in this regard are limited.

Another problem of observations in the runout zone is the rising temperature of the avalanche with its descent. The temperature increases because of dissipating kinetic energy and entrainment of warm snow (e.g., Vera Valero et al., 2015). This is not considered in the presented rheological model. The assumptions of the applied model, e.g., incompressibility and equilibrium of the granular temperature in the dense flow, are encouraged by the obtained results. Applying the microrheological parameters for snow to calculate the mean free path between collisions, we obtain $s=2 \times 10^{-4} \mathrm{~m}$. In combination with the low coefficient of restitution $\epsilon=0.1$ the granular temperature will dissipate rapidly when no additional energy is supplied, leading quickly to the assumed equilibrium. Moreover, the difference between limit states of density in the dense flow, calculated using $v_{\mathrm{rlp}}$ and $v_{\mathrm{s}}$ found for snow, is about $4 \%$, which indicates an almost incompressible flow. The validity of a steady state velocity profile cannot be verified. However, applying prescribed velocity and pressure profiles is the core feature of all depth-averaged models.

\section{Outlook}

For future works we suggest to include the energy conservation into the dynamic flow model to track the evolution of the thermodynamic temperature. Consequently, the particle diameter can be calculated (e.g., with the relation proposed by Steinkogler et al., 2015a) and used to model the dynamic friction with respect to the diameter dependency as predicted by the extended kinetic theory,

$\tau_{\mathrm{c}} \propto d^{2}$.

This will lead to a significantly increased dynamic friction for wet snow avalanches. Assuming a particle diameter of $0.2 \mathrm{~m}$ for wet snow (Bartelt and McArdell, 2009; Steinkogler et al., 2015a), $\chi$ will decrease down to $80 \mathrm{~m}^{-1} \mathrm{~s}^{-1}$.

The possibility of a negative coefficient $\chi$ at high pressures, as proposed by the extended kinetic theory, should be further investigated. This effect cannot be described by the simplified relation and does therefore not appear in the extended kinetic theory relation with the back-calculated microscopic parameters. However, this effect may help explaining the low friction of catastrophic ice and snow avalanches (e.g., Alean, 1985).

The extended kinetic theory predicts a relation between classic flow variables (e.g., $\sigma, \dot{\gamma}$ ) and the bulk density $\rho=$ $v \rho_{\mathrm{p}}$, matching the critical state line for small shear rates. Although the dense flow regime is nearly incompressible (e.g., for the snow parameters obtained in this work, the limit states for bulk density in the dense flow regime differ by about $4 \%$ ), this relation can be exploited to extended the SavageHutter model to compressible flows, based on microrheological effects.

Moreover, the transition point between dense flow regime and purely collisional regime, where the density decreases rapidly is a meaningful result for the simulation of powder snow avalanches. We found a simplified empirical description for the transition point, when applying the microscopic material parameters for snow:

$\dot{\gamma} \approx 3.16 \sigma^{0.368}$.

This relation can be used to develop novel coupling models of dense flow and powder cloud. There are several other models simulating the coupling between dense flow and powder cloud (e.g., Sampl and Zwinger, 2004; Bartelt et al., 2016).

\section{Summary}

In summary this paper highlights the application of a rheological model based on kinetic theory to depth-averaged 
snow avalanche simulations. To combine both frameworks we employed the commonly accepted assumption of a constant velocity profile along the avalanche and during its decent. The resulting relation shows similarities to classic friction relations. The employed comparison method allowed to evaluate the different basal friction models with respect to different observation variables. Here the residual sum of squares in combination with a normalization, such that values with different physical units and orders of magnitude can be combined, allowed the comparison of the presented friction relation to the widespread Voellmy friction relation. Utilizing the new relation shows some improvements, particularly when evaluating different observation variables and multiple events.

\section{Data availability}

The underlying data are intellectual property of the BFW and its scientific partners and are not available to the public. For scientific collaboration and data usage, interested researchers are invited to get in contact with the authors.

Acknowledgements. We would like to express our gratitude to the Norwegian Geotechnical Institute (NGI; P. Gauer) and the WSL Institute for Snow and Avalanche research (SLF; B. Sovilla and P. Bartelt) for collaborative experiments at the Ryggfonn and Vallée de la Sionne test sites and also for access to the respective data and P. Bartelt for fruitful discussions. We thank M. Granig (WLV Austrian Service for Torrent and Avalanche Control) and P. Sampl (AVL LIST GmbH) for their support and cooperation regarding SamosAT. Furthermore, the authors acknowledge the financial support by the OEAW project "beyond dense flow avalanches".

Edited by: P. Bartelt

Reviewed by: two anonymous referees

\section{References}

Alam, M., Willits, J. T., Arnarson, B. Ö., and Luding, S.: Kinetic theory of a binary mixture of nearly elastic disks with size and mass disparity, Phys. Fluids, 14, 4085-4087, doi:10.1063/1.1509066, 2002.

Alean, J.: Ice avalanches: some empirical information about their formation and reach, J. Glaciology, 31, 324-333, 1985.

Ancey, C.: Plasticity and geophysical flows: a review, J. NonNewtonian Fluid, 142, 4-35, doi:10.1016/j.jnnfm.2006.05.005, 2007.

Arnarson, B. Ö. and Willits, J. T.: Thermal diffusion in binary mixtures of smooth, nearly elastic spheres with and without gravity, Phys. Fluids, 10, 1324-1328, doi:10.1063/1.869658, 1998.

Bagnold, R. A.: Experiments on a gravity-free dispersion of large solid spheres in a Newtonian fluid under shear, P. Roy. Soc. Lond. A-Mat., 225, 49-63, doi:10.1098/rspa.1954.0186, 1954.
Bagnold, R. A.: The shearing and dilatation of dry sand and the 'singing' mechanism, P. Roy. Soc. Lond. A-Mat., 295, 219-232, doi:10.1098/rspa.1966.0236, 1966.

Baker, J. L., Barker, T., and Gray, J. M. N. T.: A two-dimensional depth-averaged $\mu(\mathrm{I})$-rheology for dense granular avalanches, J. Fluid Mech., 787, 367-395, doi:10.1017/jfm.2015.684, 2015.

Bartelt, P. and Buser, O.: The relation between dilatancy, effective stress and dispersive pressure in granular avalanches, Acta Geotechnica, 11, 549-557, doi:10.1007/s11440-016-04637, 2016.

Bartelt, P. and McArdell, B. W.: Granulometric investigations of snow avalanches, J. Glaciol., 55, 829-833, doi:10.3189/002214309790152384, 2009.

Bartelt, P., Salm, L. B., and Gruberl, U.: Calculating dense-snow avalanche runout using a Voellmyfluid model with active/passive longitudinal straining, J. Glaciol., 45, 242-254, 1999.

Bartelt, P., Buser, O., and Platzer, K.: Fluctuation-dissipation relations for granular snow avalanches, J. Glaciol., 52, 631-643, doi:10.3189/172756506781828476, 2006.

Bartelt, P., Buser, O., Vera Valero, C., and Bühler, Y.: Configurational energy and the formation of mixed flowing/powder snow and ice avalanches, Ann. Glaciol., 57, 179-188, doi:10.3189/2016AoG71A464, 2016.

Berzi, D., Di Prisco, C. G., and Vescovi, D.: Constitutive relations for steady, dense granular flows, Phys. Rev. E, 84, 031301, doi:10.1103/PhysRevE.84.031301, 2011.

Boemer, A., Qi, H., and Renz, U.: Eulerian simulation of bubble formation at a jet in a two-dimensional fluidized bed, Int. J. Multiphas. Flow, 23, 927-944, doi:10.1016/S0301-9322(97)00018-9, 1997.

Bouchut, F. and Westdickenberg, M.: Gravity driven shallow water models for arbitrary topography, Commun. Math. Sci., 2, 359389, 2004.

Bovis, M. J. and Mears, A. I.: Statistical prediction of snow avalanche runout from terrain variables in Colorado, Arctic Alpine Res., 8, 115-120, doi:10.2307/1550615, 1976.

Brey, J. J., Dufty, J. W., Sub Kim, C., and Santos, A.: Hydrodynamics for granular flow at low density, Phys. Rev. E, 58, 4638-4653, doi:10.1103/PhysRevE.58.4638, 1998.

Brilliantov, N. V. and Pöschel, T.: Granular gases with impactvelocity-dependent restitution coefficient, in: Granular Gases, edited by: Pöschel, T. and Luding, S., Springer Berlin Heidelberg, 100-124, doi:10.1007/3-540-44506-4_5, 2001.

Buser, O. and Bartelt, P.: Production and decay of random kinetic energy in granular snow avalanches, J. Glaciol., 55, 3-12, doi:10.3189/002214309788608859, 2009.

Buser, O. and Bartelt, P.: An energy-based method to calculate streamwise density variations in snow avalanches, J. Glaciol., 61, 563-575, doi:10.3189/2015JoG14J054, 2015.

Campbell, C. S.: Rapid granular flows, Annu. Rev. Fluid Mech., 22, 57-90, doi:10.1146/annurev.fl.22.010190.000421, 1990.

Campbell, C. S.: Granular shear flows at the elastic limit, J. Fluid Mech., 465, 261-291, doi:10.1017/S002211200200109X, 2002.

Campbell, C. S.: Stress-controlled elastic granular shear flows, J. Fluid Mech., 539, 273-297, doi:10.1017/S0022112005005616, 2005.

Campbell, C. S.: Granular material flows - an overview, Powder Technol., 162, 208-229, doi:10.1016/j.powtec.2005.12.008, 2006. 
Carnahan, N. F. and Starling, K. E.: Equation of state for nonattracting rigid spheres, J. Chem. Phys., 51, 635-636, doi:10.1063/1.1672048, 1969.

Chialvo, S., Sun, J., and Sundaresan, S.: Bridging the rheology of granular flows in three regimes, Phys. Rev. E, 85, 021305, doi:10.1103/PhysRevE.85.021305, 2012.

Christen, M., Kowalski, J., and Bartelt, P.: RAMMS: Numerical simulation of dense snow avalanches in threedimensional terrain, Cold Reg. Sci. Technol., 63, 1-14, doi:10.1016/j.coldregions.2010.04.005, 2010.

da Cruz, F., Emam, S., Prochnow, M., Roux, J.-N., and Chevoir, F.: Rheophysics of dense granular materials: Discrete simulation of plane shear flows, Phys. Rev. E, 72, 021309, doi:10.1103/PhysRevE.72.021309, 2005.

Dent, J. D., Burrell, K. J., Schmidt, D. S., Louge, M. Y., Adams, E. E., and Jazbutis, T. G.: Density, velocity and friction measurements in a dry-snow avalanche, Ann. Glaciol., 26, 247-252, 1998.

de Saint-Venant, A. J. C. B.: Théorie du mouvement nonpermanent des eaux, avec application aux crues des rivières et à l'introduction de marées dans leurs lits, CR Hebd. Acad. Sci., 73, 147-154, 1871.

Dutto, P.: Modelling of landslides propagation with SPH: effects of rheology and pore water pressure, $\mathrm{PhD}$ thesis, Universidad Politecnica De Madrid, http://oa.upm.es/33166/ (last access: 26 October 2016), 2014.

Fischer, J.-T.: A novel approach to evaluate and compare computational snow avalanche simulation, Nat. Hazards Earth Syst. Sci., 13, 1655-1667, doi:10.5194/nhess-13-1655-2013, 2013.

Fischer, J.-T., Kowalski, J., and Pudasaini, S. P.: Topographic curvature effects in applied avalanche modeling, Cold Reg. Sci. Technol., 74, 21-30, doi:10.1016/j.coldregions.2012.01.005, 2012.

Fischer, J.-T., Fromm, R., Gauer, P., and Sovilla, B.: Evaluation of probabilistic snow avalanche simulation ensembles with Doppler radar observations, Cold Reg. Sci. Technol., 97, 151158, doi:10.1016/j.coldregions.2013.09.011, 2014.

Fischer, J.-T., Kofler, A., Wolfgang, F., Granig, M., and Kleemayr, K.: Multivariate parameter optimization for computational snow avalanche simulation, J. Glaciol., 875-888, doi:10.3189/2015JoG14J168, 2015.

Forterre, Y. and Pouliquen, O.: Flows of dense granular media, Annu. Rev. Fluid Mech., 40, 1-24, doi:10.1146/annurev.fluid.40.111406.102142, 2008.

Garzó, V. and Dufty, J. W.: Dense fluid transport for inelastic hard spheres, Phys. Rev. E, 59, 5895-5911, doi:10.1103/PhysRevE.59.5895, 1999.

Garzó, V. and Dufty, J. W.: Hydrodynamics for a granular binary mixture at low density, Phys. Fluids, 14, 1476-1490, doi:10.1063/1.1458007, 2002.

Gauer, P., Kern, M., Kristensen, K., Lied, K., Rammer, L., and Schreiber, H.: On pulsed Doppler radar measurements of avalanches and their implication to avalanche dynamics, Cold Reg. Sci. Technol., 50, 55-71, doi:10.1016/j.coldregions.2007.03.009, 2007.

Gauer, P., Issler, D., Lied, K., Kristensen, K., and Sandersen, F.: On snow avalanche flow regimes: inferences from observations and measurements, in: Proceedings Whistler 2008 International Snow Science Workshop, 21-27 September 2008, 717 pp., 2008.
Goldhirsch, I.: Rapid granular flows, Annu. Rev. Fluid Mech., 35, 267-293, doi:10.1146/annurev.fluid.35.101101.161114, 2003.

Goldshtein, A. and Shapiro, M.: Mechanics of collisional motion of granular materials. Part 1. General hydrodynamic equations, J. Fluid Mech., 282, 75-114, doi:10.1017/S0022112095000048, 1995.

Gruber, U., Bartelt, P., and Margreth, S.: Anleitung zur Berechnung von Fließlawinen: Neue Berechnungsmethoden in der Lawinengefahrenkartierung, WSL Institut für Schnee-und Lawinenforschung SLF, Davos, 1999.

Hogg, A. J. and Pritchard, D.: The effects of hydraulic resistance on dam-break and other shallow inertial flows, J. Fluid Mech., 501, 179-212, doi:10.1017/S0022112003007468, 2004.

Hutter, K., Wang, Y., and Pudasaini, S. P.: The Savage-Hutter avalanche model: how far can it be pushed?, Philos. T. Roy. Soc. A, 363, 1507-1528, doi:10.1098/rsta.2005.1594, 2005.

Issler, D. and Gauer, P.: Exploring the significance of the fluidized flow regime for avalanche hazard mapping, Ann. Glaciol., 49, 193-198, doi:10.3189/172756408787814997, 2008.

Issler, D., Harbitz, C., Kristensen, K., Lied, K., Moe, A., Barbolini, M., De Blasio, F., Khazaradze, G., McElwaine, J., Mears, A., Naaim, M., and Sailer, R.: A comparison of avalanche models with data from dry-snow avalanches at Ryggfonn, Norway, in: Proc. 11th Intl. Conference and Field Trip on Landslides, Norway, Taylor Francis Ltd, 173-179, 2005.

Iverson, R. M. and George, D. L.: A depth-averaged debrisflow model that includes the effects of evolving dilatancy. I. Physical basis, P. Roy. Soc. Lond. A Mat., 470, 20130819, doi:10.1098/rspa.2013.0819, 2014.

Jenkins, J. T.: Dense shearing flows of inelastic disks, Phys. Fluids, 18, 103307, doi:10.1063/1.2364168, 2006.

Jenkins, J. T.: Dense inclined flows of inelastic spheres, Granul. Matter, 10, 47-52, doi:10.1007/s10035-007-0057-z, 2007.

Jenkins, J. T. and Berzi, D.: Dense inclined flows of inelastic spheres: tests of an extension of kinetic theory, Granul. Matter, 12, 151-158, doi:10.1007/s10035-010-0169-8, 2010.

Jenkins, J. T. and Mancini, F.: Balance laws and constitutive relations for plane flows of a dense, binary mixture of smooth, nearly elastic, circular disks, J. Appl. Mech., 54, 27-34, doi:10.1115/1.3172990, 1987.

Jenkins, J. T. and Mancini, F.: Kinetic theory for binary mixtures of smooth, nearly elastic spheres, Phys. Fluids A-Fluid, 1, 20502057, doi:10.1063/1.857479, 1989.

Jenkins, J. T. and Richman, M. W.: Grad's 13-moment system for a dense gas of inelastic spheres, in: The Breadth and Depth of Continuum Mechanics: A Collection of Papers Dedicated to J. L. Ericksen on His Sixtieth Birthday, Springer Berlin Heidelberg, 647-669, doi:10.1007/978-3-642-61634-1_31, 1986.

Jenkins, J. T. and Zhang, C.: Kinetic theory for identical, frictional, nearly elastic spheres, Phys. Fluids, 14, 1228-1235, doi:10.1063/1.1449466, 2002.

Jóhannesson, T., Gauer, P., Issler, P., and Lied, K.: The design of avalanche protection dams. Recent practical and theoretical developments, No. EUR 23339 in Climate Change and Natural Hazard Research Series 2, 2009.

Kern, M., Bartelt, P., Sovilla, B., and Buser, O.: Measured shear rates in large dry and wet snow avalanches, J. Glaciol., 55, 327338, doi:10.3189/002214309788608714, 2009. 
Körner, H. J.: Modelle zur Berechnung der Bergsturz- und Lawinenbewegung, Interpraevent 1980, 2, 15-55, 1980.

Lee, C.-H. and Huang, C.-J.: Model of sheared granular material and application to surface-driven granular flows under gravity, Phys. Fluids, 22, 043307, doi:10.1063/1.3400203, 2010.

Lois, G., Lemaître, A., and Carlson, J. M.: Numerical tests of constitutive laws for dense granular flows, Phys. Rev. E, 72, 051303, doi:10.1103/PhysRevE.72.051303, 2005.

Lois, G., Lemaitre, A., and Carlson, J. M.: Emergence of multi-contact interactions in contact dynamics simulations of granular shear flows, EPL-Europhys. Lett., 76, 318-324, doi:10.1209/epl/i2005-10605-1, 2006.

Lun, C. K. K.: Kinetic theory for granular flow of dense, slightly inelastic, slightly rough spheres, J. Fluid Mech., 233, 539-559, doi:10.1017/S0022112091000599, 1991.

Lun, C. K. K. and Savage, S. B.: A simple kinetic theory for granular flow of rough, inelastic, spherical particles, J. Appl. Mech., 54, 47-53, doi:10.1115/1.3172993, 1987.

McClung, D. M. and Schaerer, P. A.: Characteristics of flowing snow and avalanche impact pressures, Ann. Glaciol., 6, 9-14, 1985.

Mergili, M., Schratz, K., Ostermann, A., and Fellin, W.: Physicallybased modelling of granular flows with Open Source GIS, Nat. Hazards Earth Syst. Sci., 12, 187-200, doi:10.5194/nhess-12187-2012, 2012.

Mills, P., Rognon, P. G., and Chevoir, F.: Rheology and structure of granular materials near the jamming transition, EPL-Europhys. Lett., 81, 64005, doi:10.1209/0295-5075/81/64005, 2008.

Mitarai, N. and Nakanishi, H.: Bagnold scaling, density plateau, and kinetic theory analysis of dense granular flow, Phys. Rev. Lett., 94, 128001, doi:10.1103/PhysRevLett.94.128001, 2005.

Mitarai, N. and Nakanishi, H.: Velocity correlations in dense granular shear flows: Effects on energy dissipation and normal stress, Phys. Rev. E, 75, 031305, doi:10.1103/PhysRevE.75.031305, 2007.

Norem, H., Irgens, F., and Schieldrop, B.: A continuum model for calculating snow avalanche velocities, in: Proceedings of the Symposium on Avalanche Formation, Movement and Effects, 363-379, 14-19 September 1969, Davos, Switzerland, 14-19, 1987.

Patra, A. K., Bauer, A., Nichita, C., Pitman, E. B., Sheridan, M., Bursik, M., Rupp, B., Webber, A., Stinton, A., Namikawa, L., and Renschler, C. S.: Parallel adaptive numerical simulation of dry avalanches over natural terrain, J. Volcanol. Geoth. Res., 139, 1-21, doi:10.1016/j.jvolgeores.2004.06.014, 2005.

Pitman, E. B., Nichita, C. C., Patra, A., Bauer, A., Sheridan, M., and Bursik, M.: Computing granular avalanches and landslides, Phys. Fluids, 15, 3638-3646, doi:10.1063/1.1614253, 2003.

Pöschel, T. and Buchholtz, V.: Molecular dynamics of arbitrarily shaped granular particles, J. Phys. I, 5, 1431-1455, doi:10.1051/jp1:1995208, 1995.

Pouliquen, O.: Scaling laws in granular flows down rough inclined planes, Phys. Fluids, 11, 542-548, doi:10.1063/1.869928, 1999.

Pouliquen, O. and Forterre, Y.: A non-local rheology for dense granular flows, Philos. T. Roy. Soc. A, 367, 5091-5107, doi:10.1098/rsta.2009.0171, 2009.

Press, W. H., Flannery, B. P., Teukolsky, S. A., and Vetterling, W. T.: Numerical Recipes in C, Second Edition, Cambridge Univ. Press, 2002.
Rahaman, M. F., Naser, J., and Witt, P. J.: An unequal granular temperature kinetic theory: description of granular flow with multiple particle classes, Powder Technol., 138, 82-92, doi:10.1016/j.powtec.2003.08.050, 2003.

Rauter, M.: Entwicklung eines neuen Sohlreibungsmodells für Lawinen, Master's thesis, Universität Innsbruck, Innsbruck, Austria, http://resolver.obvsg.at/urn:nbn:at:at-ubi:1-3875 (last access: 26 October 2016), 2015.

Rognon, P. G., Chevoir, F., Bellot, H., Ousset, F., Naaim, M., and Coussot, P.: Rheology of dense snow flows: Inferences from steady state chute-flow experiments, J. Rheol., 52, 729-748, doi:10.1122/1.2897609, 2008.

Roscoe, K. H., Schofield, A. N., and Wroth, C. P.: On the yielding of soils, Géotechnique, 8, 22-53, doi:10.1680/geot.1958.8.1.22, 1958.

Salm, B.: Contribution to avalanche dynamics, in: Scientific Aspects of Snow and Ice Avalanches, Symposium at Davos, 510 April 1965, 69, 199-214, 1966.

Salm, B.: Flow, flow transition and runout distances of flowing avalanches, Ann. Glaciology, 18, 221-226, 1993.

Salm, B., Gubler, H. U., and Burkard, A.: Berechnung von Fliesslawinen: eine Anleitung für Praktiker mit Beispielen, WSL Institut für Schnee-und Lawinenforschung SLF, Davos, 1990.

Sampl, P. and Zwinger, T.: Avalanche simulation with SAMOS, Ann. Glaciol., 38, 393-398, doi:10.3189/172756404781814780, 2004.

Savage, S. B. and Hutter, K.: The motion of a finite mass of granular material down a rough incline, J. Fluid Mech., 199, 177-215, doi:10.1017/S0022112089000340, 1989.

Savage, S. B. and Hutter, K.: The dynamics of avalanches of granular materials from initiation to runout. Part I: Analysis, Acta Mech., 86, 201-223, doi:10.1007/BF01175958, 1991.

Scapozza, C.: Entwicklung eines dichte- und temperaturabhängigen Stoffgesetzes zur Beschreibung des visko-elastischen Verhaltens von Schnee, $\mathrm{PhD}$ thesis, Swiss Fed. Inst. of Technol., Zurich, doi:10.3929/ethz-a-004680249, 2004.

Scheidegger, A. E.: On the prediction of the reach and velocity of catastrophic landslides, Rock Mech. Rock Eng., 5, 231-236, doi:10.1007/BF01301796, 1973.

Schofield, A. and Wroth, P.: Critical state soil mechanics, 1968.

Sela, N. and Goldhirsch, I.: Hydrodynamic equations for rapid flows of smooth inelastic spheres, to Burnett order, J. Fluid Mech., 361, 41-74, doi:10.1017/S0022112098008660, 1998.

Serero, D., Goldhirsch, I., Noskowicz, S. H., and Tan, M.-L.: Hydrodynamics of granular gases and granular gas mixtures, J. Fluid Mech., 554, 237-258, doi:10.1017/S0022112006009281, 2006.

Silbert, L. E.: Jamming of frictional spheres and random loose packing, Soft Mater., 6, 2918-2924, doi:10.1039/C001973A, 2010.

Silbert, L. E., Ertaş, D., Grest, G. S., Halsey, T. C., Levine, D., and Plimpton, S. J.: Granular flow down an inclined plane: Bagnold scaling and rheology, Phys. Rev. E, 64, 051302, doi:10.1103/PhysRevE.64.051302, 2001.

Sovilla, B.: Field experiments and numerical modelling of mass entrainment and deposition processes in snow avalanches, $\mathrm{PhD}$ thesis, Swiss Fed. Inst. of Technol., Zurich, doi:10.3929/ethz-a00478484, 2004.

Sovilla, B., Burlando, P., and Bartelt, P.: Field experiments and numerical modeling of mass entrainment in snow avalanches, 
J. Geophys. Res., 111, F03007, doi:10.1029/2005JF000391, 2006.

Sovilla, B., McElwaine, J. N., and Louge, M. Y.: The structure of powder snow avalanches, C. R. Phys., 16, 97-104, doi:10.1016/j.crhy.2014.11.005, 2015.

Steinkogler, W., Gaume, J., Löwe, H., Sovilla, B., and Lehning, M.: Granulation of snow: From tumbler experiments to discrete element simulations, J. Geophys. Res.-Earth, 120, 1107-1126, doi:10.1002/2014JF003294, 2015a.

Steinkogler, W., Sovilla, B., and Lehning, M.: Thermal energy in dry snow avalanches, The Cryosphere, 9, 1819-1830, doi:10.5194/tc-9-1819-2015, 2015b.

Syamlal, M., Rogers, W., and O'Brien, T. J.: MFIX documentation: Theory guide, National Energy Technology Laboratory, Department of Energy, Technical Note DOE/METC-95/1013 and NTIS/DE95000031, 1993.

Tiefenbacher, F. and Kern, M. A.: Experimental devices to determine snow avalanche basal friction and velocity profiles, Cold Reg. Sci. Technol., 38, 17-30, doi:10.1016/S0165232X(03)00060-0, 2004.

Vallet, J., Gruber, U., and Dufour, F.: Photogrammetric avalanche volume measurements at Vallée de la Sionne, Switzerland, Ann. Glaciol., 32, 141-146, doi:10.3189/172756401781819689, 2001.

van Wachem, B. G. M.: Derivation, implementation, and validation of computer simulation models for gas-solid fluidized beds, PhD thesis, TU Delft, Delft University of Technology, Delft, the Netherlands, http://repository.tudelft.nl/assets/uuid: 919e2efa-5db2-40e6-9082-83b1416709a6/as_wachem_ 20000918.PDF (last access: 26 October 2016), 2000.

van Wachem, B. G. M., Schouten, J. C., Krishna, R., and van den Bleek, C. M.: Eulerian simulations of bubbling behaviour in gas-solid fluidised beds, Comput. Chem. Eng., 22, 299-306, doi:10.1016/S0098-1354(98)00068-4, 1998. van Wachem, B. G. M., Schouten, J. C., Krishna, R., and van den Bleek, C. M.: Validation of the Eulerian simulated dynamic behaviour of gas-solid fluidised beds, Chem. Eng. Sci., 54, 21412149, doi:10.1016/S0009-2509(98)00303-0, 1999.

Vera Valero, C., Wikstroem Jones, K., Bühler, Y., and Bartelt, P.: Release temperature, snow-cover entrainment and the thermal flow regime of snow avalanches, J. Glaciol., 61, 173-184, doi:10.3189/2015JoG14J117, 2015.

Vescovi, D.: Granular shear flows: constitutive modeling and numerical simulations, PhD thesis, Politecnico di Milano, Milan, Italy, http://hdl.handle.net/10589/89847 (last access: 26 October 2016), 2014.

Vescovi, D., di Prisco, C., and Berzi, D.: From solid to granular gases: the steady state for granular materials, Int. J. Numer. Anal. Met., 37, 2937-2951, doi:10.1002/nag.2169, 2013.

Voellmy, A.: Über die Zerstörungskraft von Lawinen, Schweizerische Bauzeitung, 73, 212-217, doi:10.5169/seals-61891, 1955.

Willits, J. T. and Arnarson, B. Ö.: Kinetic theory of a binary mixture of nearly elastic disks, Phys. Fluids, 11, 3116-3122, doi:10.1063/1.870169, 1999.

Zamankhan, P.: Kinetic theory of multicomponent dense mixtures of slightly inelastic spherical particles, Phys. Rev. E, 52, 4877, doi:10.1103/PhysRevE.52.4877, 1995.

Zwinger, T., Kluwick, A., and Sampl, P.: Numerical Simulation of Dry-Snow Avalanche Flow over Natural Terrain, in: Dynamic Response of Granular and Porous Materials under Large and Catastrophic Deformations, edited by: Hutter, K. and Kirchner, N., Springer Berlin Heidelberg, Lecture Notes in Applied and Computational Mechanics, 11, 161-194, doi:10.1007/9783-540-36565-5_5, 2003. 